\title{
Sommes de diviseurs et structure multiplicative des entiers
}

par

J.-M. Deshouillers et F. Dress (Talence)

A Paul Erdös

en hommage cordial et respectueux

1. Contexte général et énoncé du théorème principal. La résolution du problème de Waring pour les bicarrés, effectuée en collaboration par $R$. Balasubramanian et les deux auteurs de ce travail, et présentée en [1], repose sur deux résultats.

Un résultat asymptotique: tout entier supérieur à $10^{367}$ est somme de dix-neuf puissances quatrièmes.

Un résultat "fini": tout entier inférieur à $10^{378}$ est somme de dix-neuf puissances quatrièmes.

Le résultat asymptotique s'articule lui-même en quatre parties, présentées en [2], dont le présent article constitue la quatrième.

La mise en oeuvre, dans la méthode du cercle, de l'inégalité de Hua améliorée conduit à introduire une somme du type

$$
\sum_{h_{1}, h_{2}, y} d_{3}\left(h_{1} h_{2} P_{2}\left(h_{1}, h_{2}, y\right)\right)
$$

où $d_{3}(n)$ est le nombre de manières d'écrire l'entier $n$ comme produit de 3 entiers positifs, $P_{2}$ est un polynôme de degré 2 , et $h_{1}, h_{2}, y$, parcourent des intervalles de même taille. L'objet du présent article est de majorer cette somme de fonctions de diviseurs. En raison de ses aspects extrêmement calculatoires, nous commencerons par présenter l'ordonnancement de sa démonstration.

La première étape consiste à séparer les variables; pour cela, on peut utiliser la sous-multiplicativité de la fonction $d_{3}$

$$
d_{3}(a b c) \leqslant d_{3}(a) d_{3}(b) d_{3}(c)
$$

mais cela introduit une perte assez importante lorsque $a, b$ et $c$ ne sont pas premiers entre eux deux à deux. A titre d'exemple, on notera que l'on a

$$
d_{3}(16 a b c) \leqslant \frac{5}{18} d_{3}(4 a) d_{3}(2 b) d_{3}(2 c) .
$$


Ce type d'amélioration à la sous-multiplicativité de $d_{3}$ est développé dans le second paragraphe. La considération des seuls facteurs premiers 2 et 3 a constitué le meilleur compromis entre la qualité des constantes et la complication des calculs.

Les variables étant séparées, le problème se ramène à la majoration des sommes

$$
\sum_{h=r(\bmod 6)} d_{3}(h) \quad \text { et } \quad \sum_{y} d_{3}\left(Q_{2}(y)\right)
$$

où $Q_{2}$ est un polynôme quadratique irréductible. La première somme est évaluée dans le troisième paragraphe.

Plus intéressante est la seconde somme. Il a été montré en 1952 par P. Erdös [5] que l'on a

$$
\sum_{y \leqslant Y} d_{3}\left(Q_{2}(y)\right) \ll Y \log Y
$$

mais nous ne voyons pas comment obtenir une constante raisonnable dans cette majoration. On peut également essayer d'utiliser le travail [3] de van der Corput, qui avait démontré en 1939 la majoration

$$
\sum_{y \leqslant Y} d_{3}\left(Q_{2}(y)\right) \ll Y(\log Y)^{k}
$$

pour un $k$ convenable; cette fois-ci, le problème est non seulement d'obtenir une bonne constante, mais aussi un bon exposant, ce qui semble exclu par la méthode de van der Corput. C'est pour ces différentes raisons que le premier auteur [4] avait eu recours à l'inégalité de Cauchy-Schwarz pour séparer les variables.

La méthode que nous développons dans le quatrième paragraphe a bénéficié des suggestions de H. L. Montgomery et de G. Tenenbaum, que nous tenons à remercier ici. Elle part d'une nouvelle démonstration du théorème de van der Corput, donnée par D. Wolke en 1972 [9] basée sur des majorations du type

$$
d(n) \leqslant_{x}\left(\sum_{\substack{\delta \mid n \\ \delta \leqslant n^{x}}} d(\delta)\right)^{k}
$$

pour $x>0$ et pour un exposant $k=k(x)$ convenable. Dans le paragraphe 4 , nous montrons comment établir des majorations du type

$$
f(n) \leqslant C \sum_{\substack{d \mid n \\ d \leqslant \sqrt{n}}} g(d)
$$

pour une fonction multiplicative $f$ à valeurs positives. Puis, pour le cas de la fonction $d_{3}$ qui nous intéresse, une fonction efficace $g$ et une constante effective $C$ sont déterminées (proposition 4.2). Mentionnons ici qu’à la suite de notre travail, B. Landreau a étudié les inégalités plus générales du type

$$
f(n) \leqslant C \sum_{\substack{d ! n \\ d \leqslant n^{z}}} g(d)
$$

ainsi que leur application à une démonstration encore plus simple du théorème de van der Corput.

En utilisant la proposition 4.2 , on ramène alors la majoration de la somme $\sum_{y \leqslant Y} d_{3}\left(Q_{2}(y)\right)$ à celle des sommes $\sum_{y \leqslant Y} \sum_{\substack{\delta \mid Q_{2}(y) \\ \delta \leqslant j Y}} g(\delta)$; une interversion de sommations ramène notre majoration à celle de

$$
\sum_{\delta \leqslant \lambda Y} g(\delta) \sum_{\substack{\left.y \leqslant Y \\ O_{2}(y) \geqslant 0 \text { (mind } i\right)}} 1
$$

soit. finalement, à la majoration du nombre de solutions de congruences quadratiques. Ce travail, routinier mais délicat, est mené à bien dans le cinquième paragraphe.

Le problème est alors réduit à la sommation de fonctions multiplicatives; dans le sixième paragraphe, on achève la démonstration du résultat principal de cette étude, qui est la proposition 3.2 de [2].

THÉoREME (proposition 3.2 de [2]). Soient $P$ un entier, $\varepsilon=0$ ou 1 , et $H_{\varepsilon}(P)$ la somme

$$
2 \sum_{h_{1}, h_{2}, y} d_{3}\left(\left|h_{1} h_{2}\left(3\left(2 y+\varepsilon+h_{1}+h_{2}\right)^{2}+h_{1}^{2}+h_{2}^{2}\right)\right|\right)
$$

étendue aux triplets $\left(h_{1}, h_{2}, y\right)$ tels que

$$
\begin{gathered}
h_{1} h_{2} \neq 0, \\
\left|h_{1}\right|+\left|h_{2}\right| \leqslant P, \\
2 P<2 y+\varepsilon \leqslant 4 P, \\
2 P<2 y+\varepsilon+h_{1}+h_{2} \leqslant 4 P .
\end{gathered}
$$

Pour $P \geqslant 10^{80}$, on a la majoration

$$
H_{\varepsilon}(P) \leqslant C_{\varepsilon} P^{3}(\log P)^{9}(\log \log P)^{2},
$$

avec $C_{0}=0,01734$, et $C_{1}=0,818 \cdot 10^{-3}$

Remarque. Ce résultat étant ultimement destiné à étudier le nombre de représentations d'un entier $N$ en sommes de puissances quatrièmes, signalons que le paramètre $P$ sera de l'ordre de grandeur de $N^{1 / 4}$, et que la présence de $\varepsilon$ est liée à des questions de parité des puissances quatrièmes. Enfin, le choix de $10^{80}$ ne résulte d'aucun impératif "local" et correspond seulement à une cohérence globale dans la démonstration de $g(4)=19$. 
2. Réduction préliminaire. En utilisant linégalité triviale $d_{3}(a b c) \leqslant d_{3}(a) d_{3}(b) d_{3}(c)$ on peut écrire

$$
H_{\varepsilon}(P) \leqslant 2 \sum_{h_{1}} d_{3}\left(\left|h_{1}\right|\right) \sum_{h_{2}} d_{3}\left(\left|h_{2}\right|\right) \sum_{y} d_{3}\left(3\left(2 y+\varepsilon+h_{1}+h_{2}\right)^{2}+h_{1}^{2}+h_{2}^{2}\right)
$$

et l'on s'aperçoit alors que l'on peut améliorer notablement la constante numérique des majorations de $\sum_{y} d_{3}\left(3\left(2 y+\varepsilon+h_{1}+h_{2}\right)^{2}+h_{1}^{2}+h_{2}^{2}\right)$ en "localisant" $h_{1}$ et $h_{2}$ dans des progressions modulo $M=2^{\alpha} 3^{\beta}$. Bien entendu, la complication des calculs augmente très vite avec la taille de $M$ cependant que lamélioration progresse peu. Le choix $M=6$ offre un compromis efficace.

Proposmron 2.1. Pour $r_{1}$ et $r_{2}$ deux restes modulo 6 , on désigne par $M_{\varepsilon}\left(r_{1}, r_{2}\right)$ un majorant, pour $h_{1} h_{2} \neq 0,\left|h_{1}\right|+\left|h_{2}\right| \leqslant P$, et $h_{i} \equiv r_{i}(\bmod 6)$, de la somme

$$
\sum_{\substack{2 P<y+\varepsilon \leqslant 4 P \\ 2 P<2 y+\varepsilon+h_{1}+h_{2} \leqslant 4 P}} d_{3}\left(3\left(2 y+\varepsilon+h_{1}+h_{2}\right)^{2}+h_{1}^{2}+h_{2}^{2}\right) .
$$

On a alors la majoration

$$
H_{\varepsilon}(P) \leqslant 8 \sum_{\substack{r_{1} \bmod 6 \\ r_{2} \bmod 6}} \gamma_{\varepsilon}\left(r_{1}, r_{2}\right) \sum_{\substack{0<k_{i} \leqslant P \\ 0<k_{1}+k_{2} \leqslant P \\ k_{i} \equiv r_{i}(\bmod 6)}} d_{3}\left(k_{1}\right) d_{3}\left(k_{2}\right) M_{\varepsilon}\left(r_{1}, r_{2}\right),
$$

\begin{tabular}{|c|c|c|c|c|}
\hline \multicolumn{5}{|c|}{$\gamma_{0}\left(r_{1}, r_{2}\right)$} \\
\hline$r$ & 0 & 1 ou 5 & 2 ou 4 & 3 \\
\hline 0 & $25 / 243$ & $5 / 9$ & $5 / 18$ & $50 / 243$ \\
\hline 1 ou 5 & $5 / 9$ & 1 & $5 / 9$ & 1 \\
\hline 2 ou 4 & $5 / 18$ & $5 / 9$ & $5 / 18$ & $5 / 9$ \\
\hline 3 & $50 / 243$ & 1 & $5 / 9$ & $10 / 27$ \\
\hline
\end{tabular}

\begin{tabular}{|c|c|c|c|c|}
\hline \multicolumn{5}{|c|}{$\gamma_{1}\left(r_{1}, r_{2}\right)$} \\
\hline$r_{1} \quad r_{2}$ & 0 & 1 ou 5 & 2 ou 4 & 3 \\
\hline 0 & $20 / 81$ & 1 & $2 / 3$ & $10 / 27$ \\
\hline 1 ou 5 & 1 & 1 & 1. & 1 \\
\hline 2 ou 4 & $2 / 3$ & 1 & $2 / 3$ & 1 \\
\hline 3 & $10 / 27$ & 1 & 1 & $10 / 27$ \\
\hline
\end{tabular}

où les coefficients $y_{i}\left(r_{1}, r_{2}\right)$ sont donnés par les tableaux suivants
Un lemme préliminaire à cette démonstration, améliorant linégalité $d_{3}(a b c) \leqslant d_{3}(a) d_{3}(b) d_{3}(c)$, est nécessaire.

Lemme 2.2. Soient $A, B$ deux entiers positifs et $a, b$ deux diviseurs respectifs de $A$ et $B$; on $a$

$$
\frac{d_{3}(a) d_{3}(b)}{d_{3}(a b)} \leqslant \frac{d_{3}(A) d_{3}(B)}{d_{3}(A B)} .
$$

Démonstration. Par la multiplicativité de la fonction $d_{3}$, il suffit de démontrer que pour $p$ premier, $u \leqslant U$ et $v \leqslant V$, on a

$$
\frac{d_{3}\left(p^{u}\right) d_{3}\left(p^{v}\right)}{d_{3}\left(p^{u+v}\right)} \leqslant \frac{d_{3}\left(p^{U}\right) d_{3}\left(p^{V}\right)}{d_{3}\left(p^{U+V}\right)}
$$

ce qui équivaut à

$$
\frac{(u+1)(u+2)(v+1)(v+2)}{(u+v+1)(u+v+2)} \leqslant \frac{(U+1)(U+2)(V+1)(V+2)}{\left(U^{\prime}+V+1\right)(U+V+2)}
$$

cette dernière relation provient du fait que, pour $y \geqslant 0$ et $x \geqslant 0$, la fonction

$$
f(x)=\frac{(x+1)(x+2)}{(x+y+1)(x+y+2)}
$$

est croissante (séparément en $x$ et $y$ ).

Remarque. Bien entendu, ce résultat se généralise sans difficulté à une vaste classe de fonctions multiplicatives. En outre, une induction immédiate permet de l'étendre à un nombre quelconque d'entiers; ainsi dans le cas de trois entiers $A, B, C$ et leurs diviseurs $a, b, c$, on a:

$$
\begin{aligned}
\frac{d_{3}(a) d_{3}(b) d_{3}(c)}{d_{3}(a b c)} & =\frac{d_{3}(a) d_{3}(b)}{d_{3}(a b)} \cdot \frac{d_{3}(a b) d_{3}(c)}{d_{3}(a b c)} \\
& \leqslant \frac{d_{3}(A) d_{3}(B)}{d_{3}(A B)} \cdot \frac{d_{3}(A B) d_{3}(C)}{d_{3}(A B C)}=\frac{d_{3}(A) d_{3}(B) d_{3}(C)}{d_{3}(A B C)} .
\end{aligned}
$$

Pour la commodité de la réérence, nous donnons le statut de corollaire au résultat suivant où les facteurs relatifs aux nombres premiers 2 et 3 ont été isolés:

\section{Corollaire au lemme 2.2. On pose}

$$
c(x, y, z)=4 \frac{(x+y+z+1)(x+y+z+2)}{(x+1)(y+1)(z+1)(x+2)(y+2)(z+2)} .
$$

Soient ${ }^{-} A_{1}, A_{2}$ et $A_{3}$ trois entiers positifs; on suppose que $2^{u_{i}} 3^{v_{i}} \mid A_{i}$ (pour $i=1,2,3)$. On a l'inégalité

$$
d_{3}\left(A_{1} A_{2} A_{3}\right) \leqslant c\left(u_{1}, u_{2}, u_{3}\right) c\left(v_{1}, v_{2}, v_{3}\right) d_{3}\left(A_{1}\right) d_{3}\left(A_{2}\right) d_{3}\left(A_{3}\right)
$$


Démonstration de 1a proposition 2.1. On découpe la somme $H_{\varepsilon}(P)$ selon $r_{1}$ et $r_{2}$ modulo 6 , et on utilise le corollaire du lemme 2.2 pour écrire

$$
\begin{aligned}
& d_{3}\left(\left|h_{1} h_{2}\left(3\left(2 y+\varepsilon+h_{1}+h_{2}\right)^{2}+h_{1}^{2}+h_{2}^{2}\right)\right|\right) \\
& \leqslant \gamma_{\varepsilon}\left(r_{1}, r_{2} ; 2\right) \gamma_{\varepsilon}\left(r_{1}, r_{2} ; 3\right) d_{3}\left(\left|h_{1}\right|\right) d_{3}\left(\left|h_{2}\right|\right) d_{3}\left(3\left(2 y+\varepsilon+h_{1}+h_{2}\right)^{2}+h_{1}^{2}+h_{2}^{2}\right)
\end{aligned}
$$

où $\gamma_{\varepsilon}(\ldots ; 2)$ et $\gamma_{\varepsilon}(\ldots ; 3)$ correspondent aux facteurs primaires relatifs à $p=2$ et $p=3$. Les tableaux ci-dessous, qui donnent les valeurs de ces coefficients, ont été constitués en remarquant les implications suivantes (notations $\left.a=\left|h_{1}\right|, b=\left|h_{2}\right|, c=3\left(2 y+\varepsilon+h_{1}+h_{2}\right)^{2}+h_{1}^{2}+h_{2}^{2}\right):$

$$
p=2, \varepsilon=0
$$

- $r_{1}$ et $r_{2}=0$ ou 2 ou $4 \Rightarrow 2|a, 2| b, 4 \mid c$, et $c(1,1,2)=5 / 18$;

$-r_{1}=0$ ou 2 ou 4 et $r_{2}=1$ ou 3 ou $5 \Rightarrow 2|a, 4| c$ et $c(1,0,2)=5 / 9$;

- de même pour $r_{1}=1$ ou 3 ou 5 et $r_{2}=0$ ou 2 ou 4 ;

$p=2, \varepsilon=1$

$-r_{1}$ et $r_{2}=0$ ou 2 ou $4 \Rightarrow 2|a, 2| b$, et $c(1,1,0)=2 / 3$;

\begin{tabular}{|c|c|c|c|c|}
\hline$r_{2}$ & 0 & 1 ou 5 & 2 ou 4 & 3 \\
\hline 0 & $5 / 18$ & $5 / 9$ & $5 / 18$ & $5 / 9$ \\
\hline 1 ou 5 & $5 / 9$ & 1 & $5 / 9$ & 1 \\
\hline 2 ou 4 & $5 / 18$ & $5 / 9$ & $5 / 18$ & $5 / 9$ \\
\hline 3 & $5 / 9$ & 1 & $5 / 9$ & 1 \\
\hline
\end{tabular}

$p=3, \varepsilon=0$ ou 1

\begin{tabular}{|c|c|c|c|c|}
\hline \multicolumn{5}{|c|}{$\gamma_{1}\left(r_{1}, r_{2} ; 2\right)$} \\
\hline$r_{1}$ & 0 & 1 ou 5 & 2 ou 4 & 3 \\
\hline 0 & $2 / 3$ & 1 & $2 / 3$ & 1 \\
\hline 1 ou 5 & 1 & 1 & 1 & 1 \\
\hline 2 on 4 & $2 / 3$ & 1 & $2 / 3$ & 1 \\
\hline 3 & 1 & 1 & 1. & 1 \\
\hline
\end{tabular}

$-r_{1}$ et $r_{2}=0$ ou $3 \Rightarrow 3|a, 3| b, 3 \mid c$ et $c(1,1,1)=10 / 27$.

\begin{tabular}{|c|c|c|c|c|}
\hline$r_{1}$ & 0 & 1 ou 5 & 2 ou 4 & 3 \\
\hline 0 & $10 / 27$ & 1 & 1 & $10 / 27$ \\
\hline 1 ou 5 & 1 & 1 & 1 & 1 \\
\hline 2 ou 4 & 1 & 1 & 1 & 1 \\
\hline 3 & $10 / 27$ & 1 & 1 & $10 / 27$ \\
\hline
\end{tabular}

$\gamma_{c}\left(r_{1}, r_{2} ; 3\right)(\varepsilon=0$ ou 1 indifféremment $)$

On constate que les produits $\gamma_{\varepsilon}\left(r_{1}, r_{2} ; 2\right) \gamma_{\varepsilon}\left(r_{1}, r_{2} ; 3\right)$ sont égaux aux coefficients $\gamma_{E}\left(r_{1}, r_{2}\right)$ donnés dans l'énoncé de la proposition 2.1 et ron a donc

$$
H_{\varepsilon}(P) \leqslant 2 \sum_{\substack{r_{1} \bmod 6 \\ r_{2} \bmod 6}} \gamma_{\varepsilon}\left(r_{1}, r_{2}\right) \sum_{\substack{h_{1} h_{2} \neq 0 \\\left|h_{1}\right|+\left|h_{2}\right| \leqslant P \\ h_{i} \equiv r_{i}(\bmod 6)}} d_{3}\left(\left|h_{1}\right|\right) d_{3}\left(\left|h_{2}\right|\right) M_{\varepsilon}\left(r_{1}, r_{2}\right)
$$

Pour obtenir la formule (2.1), il reste à remarquer que linvariance des coefficients $\gamma_{\varepsilon}\left(r_{1}, r_{2}\right)$ par $r_{1} \mapsto-r_{1}$ et $r_{2} \mapsto-r_{2}(\bmod 6)$ permet de remplacer chaque somme

$$
\sum_{\substack{h_{1} h_{2} \neq 0 \\\left|h_{1}+\right| h_{2} \mid \leqslant P \\ h_{i} \equiv r_{i}(\bmod 6)}} d_{3}\left(h_{1}\right) d_{3}\left(h_{2}\right) \quad \text { par } \quad 4 \sum_{\substack{0<k_{i} \leqslant P \\ 0<k_{1}+k_{2} \leqslant P \\ k_{i}} r_{i}(\bmod 6)} d_{3}\left(k_{1}\right) d_{3}\left(k_{2}\right) .
$$

La majoration de $H_{\varepsilon}(P)$ est ainsi ramenée à des majorations des sommes $\sum_{0} d_{3}(k)$ et à des estimations des majorants $M_{\varepsilon}\left(r_{1}, r_{2}\right)$. $\underset{\substack{0 \\ k=k}(\bmod 6)}{\bmod }$

3. Sommation des fonctions $d_{2}$ et $d_{3}$ dans des progressions arithmétiques

3a. Sommation de $d_{2}$. On utilisera la fonction $i$ qui vaut 1 pour tout entier positif avec la convention traditionnelle: $i(x)=0$ si $x \in R-N^{*}$.

On rappelle l'estimation classique, valable pour tout $X \geqslant 1$ :

$$
\left|\sum_{n \leqslant X} \frac{1}{n}-\log X-\gamma\right|<\frac{7}{12 X}
$$

On introduit maintenant les fonctions multiplicatives $\chi_{r}$ et $\tau_{r}$, où $r$ parcourt l'ensemble $\{0,1, \ldots, 5\}$ des restes modulo 6 :

$$
\begin{gathered}
\chi_{r}(n)= \begin{cases}1 & \text { si }(n, 6) \mid r, \\
0 & \text { sinon, }\end{cases} \\
\tau_{r}(n)=(n, 6) \chi_{r} .
\end{gathered}
$$


On donne ci-dessous le tableau des valeurs des fonctions $\tau_{r}$ :

\begin{tabular}{|c|cccccc|}
\hline$n(\bmod 6)$ & 0 & 1 & 2 & 3 & 4 & 5 \\
\hline$\tau_{0}$ & 6 & 1 & 2 & 3 & 2 & 1 \\
$\tau_{1}, \tau_{5}$ & 0 & 1 & 0 & 0 & 0 & 1 \\
$\tau_{2}, \tau_{4}$ & 0 & 1 & 2 & 0 & 2 & 1 \\
$\tau_{3}$ & 0 & 1 & 0 & 3 & 0 & 1 \\
\hline
\end{tabular}

On introduit enfin les fonctions sommatoires

$$
T_{r}(X)=\sum_{n \leqslant X} \tau_{r}(n) \quad \text { et } \quad t_{r}(X)=\sum_{n \leqslant X} \tau_{r}(n) / n
$$

Lemme 3.1. On a les formules et, pour tout $X \geqslant 1$, les estimations suivantes:

$$
\left\{\begin{aligned}
\tau_{0}(n) & =i(n)+i(n / 2)+2 i(n / 3)+2 i(n / 6), \\
\tau_{1}(n), \tau_{5}(n) & =i(n)-i(n / 2)-i(n / 3)+i(n / 6), \\
\tau_{2}(n), \tau_{4}(n) & =i(n)+i(n / 2)-i(n / 3)-i(n / 6), \\
\tau_{3}(n) & =i(n)-i(n / 2)+2 i(n / 3)-2 i(n / 6) ;
\end{aligned}\right.
$$

$$
\begin{gathered}
\left|T_{0}(X)-\left(\frac{5}{2} X-3\right)\right| \leqslant 3, \\
\left|T_{1}(X)-\frac{1}{3} X\right| \leqslant 2 / 3, \\
\left|T_{2}(X)-X\right| \leqslant 1 \\
\left|T_{3}(X)-\frac{5}{6} X\right| \leqslant 3 / 2, \\
\mid T_{4}(X) \ldots\left(\text { comme pour } T_{2}\right) \ldots, \\
\mid T_{5}(X) \ldots\left(\text { comme pour } T_{1}\right) \ldots ; \\
\left|t_{0}(X)-\left(\frac{5}{2} \log X+\frac{5}{2} \gamma-\frac{5}{6} \log 2-\log 3\right)\right|<7 / 2 X, \\
\left|t_{1}(X)-\left(\frac{1}{3} \log X+\frac{1}{3} \gamma+\frac{1}{3} \log 2+\frac{1}{6} \log 3\right)\right|<7 / 3 X, \\
\left|t_{2}(X)-\left(\log X+\gamma-\frac{1}{3} \log 2+\frac{1}{2} \log 3\right)\right|<7 / 3 X, \\
\left|t_{3}(X)-\left(\frac{5}{6} \log X+\frac{5}{6} \gamma+\frac{5}{6} \log 2-\frac{1}{3} \log 3\right)\right|<7 / 2 X \\
\mid t_{4}(X) \ldots\left(\text { comme pour } t_{2}\right) \ldots \\
\mid t_{5}(X) \ldots\left(\text { comme pour } t_{1}\right) \ldots
\end{gathered}
$$

La démonstration des formules (3.2) peut se faire par une simple vérification finie.
Bien entendu, les fonctions $\chi_{r}$ et $\tau_{r}$ pourraient être définies de façon générale pour un module quelconque, et les formules (3.2) écrites également de façon générale. Compte tenu de la finalité strictement utilitaire du lemme 3.1, cette généralité a paru tout à fait inutile.

La fonction $\tau_{r}$ étant périodique, les formules (3.3) se contrôlent sur un intervalle de longueur 6.

Enfin, si l'on considère une formule $\tau_{r}(n)=\sum_{k} a_{k} i(n / k)$, on en déduit immédiatement

$$
t_{r}(X)=\sum_{k} \frac{a_{k}}{k} t\left(\frac{X}{k}\right)
$$

en notant $t(X)$ la fonction sommatoire $\sum_{n \leqslant X} 1 / n$. Il suffit alors d'utiliser l'estimation (3.1) de $t(X)$ pour en déduire, selon les coefficients $a_{1}, a_{2}, a_{3}$, et $a_{6}$, les estimations (3.4).

Proposition 3.2. Pour tout $X \geqslant 1$, on a les estimations

$$
\left|\sum_{\substack{n \leq X \\ n \equiv \text { r(max }(6)}} d(n)-\alpha_{r} X\left(\log X+\beta_{r}\right)\right|<\frac{31}{6} \sqrt{X}
$$

avec les valeurs suivantes des coefficients:

$$
\begin{aligned}
\alpha_{0}=\frac{15}{36}, & \beta_{0}=2 \gamma-1-\frac{2}{3} \log 2-\frac{4}{5} \log 3, \\
\alpha_{1}=\alpha_{5}=\frac{2}{36}, & \beta_{1}=\beta_{5}=2 \gamma-1+2 \log 2+\log 3, \\
\alpha_{2}=\alpha_{4}=\frac{6}{36}, & \beta_{2}=\beta_{4}=2 \gamma-1-\frac{2}{3} \log 2+\log 3, \\
\alpha_{3}=\frac{5}{36}, & \beta_{3}=2 \gamma-1+2 \log 2-\frac{4}{5} \log 3 .
\end{aligned}
$$

Corollaire. Pour tout $X \geqslant 1$, on a les estimations

$$
\left|\sum_{\substack{n \leq X \\(n, 6)=1}} d(n)-\frac{X}{9}(\log X+\beta)\right|<\frac{34}{9} \sqrt{X}
$$

avec $\beta=2 \gamma-1+2 \log 2+\log 3=2,63933 \ldots$, et

$$
\left|\sum_{\substack{n \leq X \\(n, 6)=1}} \frac{d(n)}{n}-\left(\frac{\log ^{2} X}{18}+\frac{\beta+1}{9} \log X\right)\right|<1,647 .
$$

Démonstration de la proposition 3.2. On écrit

$$
\begin{aligned}
\sum_{\substack{n \leqslant x \\
n \equiv r(\bmod 6)}} d(n) & =\sum_{\substack{n \leqslant x \\
n \equiv r(\bmod 6)}}\left(\sum_{\substack{\delta \mid n \\
\delta \leqslant \sqrt{X}}} 1+\sum_{\substack{\delta \mid n \\
\delta \geqslant n / \sqrt{X}}} 1-\sum_{\substack{\delta \mid n \\
n / \sqrt{X} \leqslant \delta \leqslant \sqrt{X}}} 1\right) \\
& =\sum_{\substack{n \leqslant X \\
n \equiv r(\bmod 6)}}\left(2 \sum_{\substack{\delta \mid n \\
\delta \leqslant \sqrt{x}}} 1-\sum_{\substack{\delta \mid n \\
n / \sqrt{X} \leqslant \delta \leqslant \sqrt{X}}} 1\right) .
\end{aligned}
$$


Il faut maintenant permuter les sommations et, pour ce faire, on considère les conditions de congruence et de divisibilité, indépendamment des contraintes de taille. On note que $\sum_{n \equiv r(\bmod 6)} \sum_{\delta \mid n}$ équivaut à $\sum_{(\delta, 6) \mid(r, 6)} \sum_{\substack{n \equiv r(\bmod 6) \\ n \equiv 0(\bmod \delta)}}$. On remarque alors, d'une part que $(\delta, 6) \mid(r, 6)$ équivaut à $(\delta, 6) \mid r$ et d'autre part que, sachant que $(\delta, 6) \mid r$ les deux conditions $n \equiv r(\bmod 6)$ et $n \equiv 0(\bmod \delta)$ équivalent à $n \equiv k(\bmod [\delta, 6])$ pour un certain $k$ (dont la détermination ne sera pas utile).

On peut donc écrire maintenant

$$
\begin{aligned}
\sum_{\substack{n \leqslant X \\
n \equiv r(\bmod 6)}} d(n) & =2 \sum_{\substack{\delta \leqslant \sqrt{X} \\
(\delta, 6) \mid r}} \sum_{\substack{n \leqslant X \\
n \equiv k(\bmod [\delta, 6])}} 1-\sum_{\substack{\delta \leqslant \sqrt{X} \\
(\delta, 6) \mid r}} \sum_{\substack{n \leqslant \delta \sqrt{X} \\
n \equiv k \bmod [\delta, 6])}} 1 \\
& =\sum_{\substack{\delta \leqslant \sqrt{X} \\
(\delta, 6)] r}} 2\left(\frac{X}{[\delta, 6]}+\vartheta\right)-\left(\frac{\delta \sqrt{X}}{[\delta, 6]}+\vartheta^{\prime}\right)
\end{aligned}
$$

avec $|\vartheta|<1$ et $\left|\vartheta^{\prime}\right|<1$. On remplace maintenant $[\delta, 6]$ par $6 \delta /(\delta, 6)$ et on utilise les fonctions $\chi_{r}$ et $\tau_{r}$ intoduites au début de ce paragraphe; on obtient

$$
\begin{aligned}
\sum_{\substack{n \leqslant X \\
n \equiv r \text { mod } 6)}} d(n)= & \sum_{\delta \leqslant \sqrt{X}}\left\{2\left(\frac{(\delta, 6)}{6 \delta} X+\vartheta\right)-\left(\frac{(\delta, 6)}{6} \sqrt{X}+\vartheta^{\prime}\right)\right\} \chi_{r}(\delta) \\
= & \frac{X}{3} \sum_{\delta \leqslant \bar{x}} \frac{\tau_{r}(\delta)}{\delta}+2 \sum_{\delta \leqslant \sqrt{X}} \vartheta \chi_{r}(\delta) \\
& -\frac{\sqrt{X}}{6} \sum_{\delta \leqslant \sqrt{X}} \tau_{r}(\delta)-\sum_{\delta \leqslant \sqrt{X}} \vartheta^{\prime} \chi_{r}(\delta) .
\end{aligned}
$$

Le terme principal $\alpha_{r} X\left(\log X+\beta_{r}\right)$ de (3.5) provient des termes principaux des estimations (3.4) de $t_{r}$ et (3.3) de $T_{r}$, reportées dans

$$
\frac{X}{3} \sum_{\delta \leqslant \sqrt{X}} \frac{\tau_{r}(\delta)}{\delta}-\frac{\sqrt{X}}{6} \sum_{\delta \leqslant \sqrt{X}} \tau_{r}(\delta)
$$

La majoration du reste est celle qui correspond à $r=0$ (la plus grande de toutes) et provient, d'une part des majorations des restes dans (3.4) et (3.3), d'autre part de

$$
\left|\sum_{\delta \leqslant \sqrt{X}}\left(2 \vartheta-\vartheta^{\prime}\right) \chi_{0}(\delta)\right| \leqslant 3 \sqrt{X}
$$

Démonstration du corollaire. L'estimation (3.6) provient tout simplement de l'addition des estimations (3.5) pour $r=1$ et $r=5$. Comme le coefficient de la majoration en $\sqrt{X}$ joue un rôle non négligeable dans la constante de majoration dans (3.7), on a repris son estimation, utilisant notamment

$$
\left|\sum_{\delta \leqslant \bar{X}}\left(2 \vartheta-\vartheta^{\prime}\right) \chi_{r}(\delta)\right| \leqslant 3\left(\frac{1}{3} \sqrt[n]{X}\right) \quad \text { si } \quad r=1 \text { ou } 5
$$

On démontre (3.7) au moyen d'une intégration par parties: on note $\Delta(x)$ $=\sum_{n \leq X} d(n)$ et on écrit $\sum_{\substack{n \leqslant X \\(n, 6)=1}}$

$$
\begin{aligned}
\sum_{\substack{n \leq X \\
(n, 6)=1}} \frac{d(n)}{n} & =\int_{1}^{x} \frac{d \Delta(t)}{t}=\left[\frac{\Delta(t)}{t}\right]_{1}^{x}+\int_{1}^{x} \frac{\Delta(t)}{t^{2}} d t \\
& =\frac{1}{9}(\log X+\beta)+\frac{34}{9}\left(\frac{\vartheta}{\sqrt{X}}\right)+\frac{1}{9} \int_{1}^{x}\left(\frac{\log t}{t}+\frac{\beta}{t}\right) d t+\frac{34}{9} \int_{1}^{X} \frac{\vartheta^{\prime}}{t^{3 / 2}} d t
\end{aligned}
$$

avec $|\vartheta|<1$ et $\left|\vartheta^{\prime}\right|<1$.

L'application brutale de cette relation fournit

$$
\left|\sum_{\substack{n \leqslant X \\(\delta, 6)=1}} \frac{d(n)}{n}-\left(\frac{\log ^{2} X}{18}+\frac{\beta+1}{9} \log X+\frac{\beta}{9}\right)\right|<\frac{68}{9}\left(1-\frac{1}{2 \sqrt{X}}\right)
$$

Mais on peut aussi sommer et intégrer de $X_{0}$ à $X$. Avec le choix $X_{0}=100$ et compte tenu des valeurs $\sum d(n) / n=3,55381 \ldots$ et

$$
b=\sum_{\substack{n \leqslant 100 \\(n, 6)=1}} \frac{d(n)}{n}-\frac{\log ^{2} 100}{18}-\frac{\beta+1}{9} \log 100=0,5134 \ldots,
$$

on obtient

$$
\left|\sum_{\substack{n \leqslant X \\(n, 6)=1}} \frac{d(n)}{n}-\left(\frac{\log ^{2} X}{18}+\frac{\beta+1}{9} \log X\right)-b\right|<\frac{34}{9}\left(\frac{3}{10}-\frac{1}{\sqrt{X}}\right),
$$

ce qui fournit la constante $1,647>0,5134 \ldots+17 / 15$ de l'estimation (3.7).

\section{3b. Sommation de $d_{3}$.}

Proposition 3.3. Pour tout $X \geqslant 1$, on a les majorations

$$
\sum_{\substack{n \leqslant X \\ n=r(\bmod 6)}} d_{3}(n)<u_{r} X\left(\log ^{2} X+v_{r} \log X+w_{r}\right)+31 \sqrt{X}
$$

avec les valeurs suivantes des coefficients:

$$
\begin{array}{rlrl}
u_{0}=\frac{133}{432}, & v_{0}=10,5268, & w_{0}=30,1432, \\
u_{1}=u_{5}=\frac{1}{108}, & v_{1}=v_{5}=19,2787, & w_{1}=w_{5}=61,3136, \\
u_{2}=u_{4}=\frac{7}{108}, & v_{2}=v_{4}=14,1359, & w_{2}=w_{4}=38,2338, \\
u_{3}=\frac{19}{432}, & . & v_{3}=12,9629, & w_{3}=36,5491 .
\end{array}
$$


Corollarre. Pour tout $X \geqslant \frac{1}{40} 10^{80}$, on a les majorations

$$
\sum_{\substack{n \leqslant X \\ n \equiv r(\bmod 6)}} d_{3}(n) \leqslant \delta(r) X \log ^{2} X
$$

avec les valeurs suivantes des coefficients:

$$
\begin{aligned}
\delta(0) & =0,32611, \\
\delta(1)=\delta(5) & =0,01027, \\
\delta(2)=\delta(4) & =0,06997, \\
\delta(3) & =0,04719 .
\end{aligned}
$$

Démonstration de la proposition 3.3. De même que l'on était parti de $d(n)$ alias $d_{2}(n)=\sum 1$, on part de $d_{3}(n)=\sum_{\delta \mid n} d(n)$. On utilise également le même procédé pour traiter la condition de congruence, et on obtient:

$$
\begin{aligned}
\sum_{\substack{n \leqslant X \\
n \equiv r(\bmod 6)}} d_{3}(n) & =\sum_{\substack{n \leqslant X \\
n \equiv r(\bmod 6)}} \sum_{\delta \mid n} d(\delta) \\
& =\sum_{\substack{\delta \leqslant X \\
(\delta, 6) \mid r}} d(\delta) \sum_{\substack{n \leqslant X \\
n \equiv k(\bmod [\delta, 6])}} 1 \\
& =\sum_{\substack{\delta \leqslant X \\
(\delta, 6\} \mid r}} d(\delta)\left(\frac{X}{[\delta, 6]}+\vartheta\right) \\
& =\sum_{\delta \leqslant X} d(\delta)\left(\frac{(\delta, 6)}{6 \delta} X+\vartheta\right) \chi_{r}(\delta),
\end{aligned}
$$

avec $|\vartheta|<1$, On en déduit la majoration

$$
\sum_{\substack{n \leqslant X \\ n \equiv r(m \text { mod } 6)}} d_{3}(n) \leqslant \frac{X}{6} \sum_{\delta \leqslant X} \frac{d(\delta)}{\delta} \tau_{r}(\delta)+\sum_{\delta \leqslant X} d(\delta) \chi_{r}(\delta)
$$

Pour éliminer le facteur $\tau_{r}(\delta)$ dans le premier terme, on se souvient qu'il s'agit d'une fonction multiplicative qui vaut 1 si $\delta$ est premier avec 6 et l'on utilise la remarque suivante, valable pour toute fonction multiplicative $g$ à valeurs réelles positives, et tout module $M=p_{1} \ldots p_{k}$ :

$$
\sum_{n \leqslant X} g(n) \leqslant \sum_{\substack{x_{1} \\ m=p_{1} \ldots p_{k}}} g(m) \sum_{\substack{n \leqslant X \\(n, M)=1}} g(n)=\prod_{i=1}^{k}\left(\sum_{\alpha=0}^{x} g\left(p_{i}^{\alpha}\right)\right) \sum_{\substack{n \leqslant X \\(n, M)=1}} g(n)
$$

(la perte dans la majoration de $\sum_{n \leqslant x} g(n)$ est d'autant plus faible que les produits infinis convergent rapidement; dans le cas présent, le terme principal sera en $X \log ^{2} X$ et la perte portera sur le terme suivant, en $X \log X$ ).
On pose alors:

$$
\begin{gathered}
F_{2}=\sum_{x=0}^{\infty} \frac{d\left(2^{x}\right)}{2^{x}}\left(2^{x}, 6\right)=1+\sum_{x=1}^{\infty} \frac{\alpha+1}{2^{x-1}}=7, \\
F_{3}=\sum_{x=0}^{\infty} \frac{d\left(3^{x}\right)}{3^{x}}\left(3^{x}, 6\right)=1+\sum_{x=1}^{\infty} \frac{\alpha+1}{3^{x-1}}=\frac{19}{4}, \\
F(r)=\sum_{m=2^{x} 3^{\beta}} \frac{d(m)}{m} \tau_{r}(m)=\left\{\begin{array}{lll}
F_{2} F_{3} & \text { si } & r=0, \\
1 & \text { si } r=1 \text { ou } 5, \\
F_{2} & \text { si } r=2 \text { ou } 4, \\
F_{3} & \text { si } r=3 .
\end{array}\right.
\end{gathered}
$$

Le second terme se découpe suivant les progressions arithmétiques modulo 6, et lon obtient finalement:

$$
\sum_{\substack{n \leqslant X \\ n \equiv r(\operatorname{mad} 6)}} d_{3}(n) \leqslant \frac{X}{6} F(r) \sum_{\substack{\delta \leqslant X \\(\delta, 6)=1}} \frac{d(\delta)}{\delta}+\sum_{\varrho=1}^{6} \chi_{r}(\varrho) \sum_{\substack{\delta \leqslant X \\ \delta \equiv \varrho(\bmod 6)}} d(\delta)
$$

On reporte les valeurs trouvées ci-dessus pour $F(r)$, et les valeurs des estimations (3.7) et (3.5), et l'on obtient les majorations de la proposition 3.3 (le terme $31 \sqrt{X}$ pourrait être amélioré, mais cela n'a aucun intérêt ici).

Démonstration du corollaire. Si $X_{0}=\frac{1}{40} 10^{80}$ et si on pose $L_{0}=\log X_{0}(=180,517 \ldots)$, on a trivialement

$$
u X\left(\log ^{2} X+v \log X+w\right)+31 \sqrt{X} \leqslant u\left(1+\frac{v}{L_{0}}+\frac{w}{L_{0}^{2}}+\frac{31}{u L_{0}^{2} \sqrt{X_{0}}}\right) X \log ^{2} X
$$

pour $X \geqslant X_{0}$.

Il faut maintenant majorer les sommes

$$
\sum_{\substack{0<k_{i} \leqslant P \\ 0<k_{1}+k_{2} \leqslant P \\ k_{i}=r_{i}(\bmod 6)}} d_{3}\left(k_{1}\right) d_{3}\left(k_{2}\right)
$$

Pour obtenir une majoration voisine de la majoration heuristiquement optimale $\frac{1}{2} \delta\left(r_{1}\right) \delta\left(r_{2}\right) X^{2} \log ^{4} X$, il est nécessaire d'utiliser le lemme suivant, qui est une version simplifiée de l'inégalité de Deshouillers-Dress-Harlipó pour les suites numériques. (Cette appellation suggère qu'il s'agit d'inégalités de réarrangement dans l'esprit du chapitre 10 de [6].)

LeMme 3.4. Soient $n$ un entier naturel et $\left(a_{i}\right),\left(a_{i}^{\prime}\right),\left(b_{i}\right),\left(b_{i}^{\prime}\right)$ quatre suites finies, avec $i \in\left\{1, \ldots, n_{\}}\right.$, de nombres réels positifs vérifiant les inégalités,

$$
\begin{array}{ll}
\forall k \in\{1, \ldots, n\} \quad & \sum_{i=1}^{\bar{k}} a_{i} \leqslant \sum_{i=1}^{k} a_{i}^{\prime}, \\
\forall k \in\{1, \ldots, n\} \quad & \sum_{i=1}^{k} b_{i} \leqslant \sum_{i=1}^{k} b_{i}^{\prime} .
\end{array}
$$


Alors on a linégalité

$$
\sum_{i+j \leqslant n} a_{i} b_{j} \leqslant \sum_{i+j \leqslant n} a_{i}^{\prime} b_{j}^{\prime}
$$

Démonstration. On fabrique une suite double finie $\left(\alpha_{i}^{h}\right)$, avec $h \in\{0, \ldots, n-1\}$ et $i \in\{1, \ldots, n\}$, de la façon suivante:

$$
\begin{aligned}
& \alpha_{i}^{0}=a_{i} \quad \text { pour } \quad 1 \leqslant i \leqslant n-1, \\
& \begin{array}{l}
\alpha_{n}^{0}=\sum_{i=1}^{n} a_{i}^{\prime}-\sum_{i=1}^{n-1} a_{i} ; \\
\alpha_{i}^{h+1}=\alpha_{i}^{h} \quad \text { pour } \quad 1 \leqslant i \leqslant n-h-2 \\
\quad \text { et } n-h+1 \leqslant i \leqslant n, \\
\alpha_{n-h-1}^{h+1}=\alpha_{n-h-1}^{h}+\alpha_{n-h}^{h}-a_{n-h}^{\prime}, \\
\alpha_{n-h}^{h+1}=a_{n-h}^{\prime} .
\end{array}
\end{aligned}
$$

De façon imagée, on commence par augmenter le dernier terme jusqu'au maximum compatible avec $\sum_{i=1}^{n} \alpha_{i}^{0} \leqslant \sum_{i=1}^{n} a_{i}^{\prime}$. Ensuite, à chaque étape $h \mapsto h+1$, on ne modifie que deux termes, ceux de rang $n-h-1$ et $n-h$ en donnant à $\alpha_{n-h}^{h+1}$ la valeur $a_{n-h}^{\prime}$ et en conservant invariante leur somme.

On vérifie alors sans difficulté un certain nombre de relations:

$$
\begin{gathered}
\forall h \in\{0, \ldots, n-1\} \forall k \in\{1, \ldots, n\} \quad \sum_{i=1}^{k} \alpha_{i}^{h} \leqslant \sum_{i=1}^{k} a_{i}^{\prime}, \\
\forall h \in\{0, \ldots, n-1\} \forall i \geqslant n-h+1 \quad \alpha_{i}^{h}=a_{i}^{\prime},
\end{gathered}
$$

la deuxième relation entraînant que les suites $\left(\alpha_{i}^{n-1}\right)$ et $\left(a_{i}^{\prime}\right)$ sont identiques. On a trivialement

$$
\sum_{i+j \leqslant n} a_{i} b_{j} \leqslant \sum_{i+j \leqslant n} \alpha_{i}^{0} b_{j} .
$$

Le procédé de formation de $\left(\alpha_{i}^{h+1}\right)$ et les inégalités $(*)$ impliquent qu'à l'étape numéro $h+1$ on a

$$
\alpha_{n-h-1}^{h+1}=\alpha_{n-h-1}^{h}+\gamma \quad \text { et } \quad \alpha_{n-h}^{h+1}=\alpha_{n-h}^{h}-\gamma
$$

avec $\gamma$ positif (rappelons que les autres termes restent inchangés). On en déduit

$$
\begin{aligned}
\sum_{i+j \leqslant n} \alpha_{i}^{h} b_{j}-\sum_{i+j \leqslant n} \alpha_{i}^{h+1} b_{j} \\
=\left(\alpha_{n-h-1}^{h}-\alpha_{n-h-1}^{h+1}\right) \sum_{i=1}^{h+1} b_{j}+\left(\alpha_{n-h}^{h}-\alpha_{n-h}^{h+1}\right) \sum_{i=1}^{h} b_{j}=-\gamma b_{h+1} \leqslant 0
\end{aligned}
$$

et il s'ensuit immédiatement l'inégalité

$$
\sum_{i+j \leqslant n} a_{i} b_{j} \leqslant \sum_{i+j \leqslant n} \alpha_{i}^{n-1} b_{j}=\sum_{i+j \leqslant n} a_{i}^{\prime} b_{j} .
$$

On effectue alors les mêmes manipulations pour $\left(b_{i}\right)$ et $\left(b_{i}^{\prime}\right)$, et la démonstration du lemme est terminée.

Remarque. À des détails de notations près, la même démonstration fonctionne en remplaçant $\sum_{i+j \leqslant n}$ par $\sum_{i=1}^{n} \sum_{j=1}^{f(i)}$, où $f$ est monotone décroissante.

Propostrion 3.5. Avec les $M_{\varepsilon}\left(r_{1}, r_{2}\right)$ définis à la proposition 2.1 , les valeurs des coefficients $\gamma_{\varepsilon}\left(r_{1}, r_{2}\right)$ données par les tableaux qui accompagnent cette proposition, et les valeurs des coefficients $\gamma(r)$ données par le tableau (3.8) $d u$ corollaire de la proposition 3.3 , on a la majoration, valable pour $P \geqslant 10^{80}$

$$
H_{\varepsilon}(P)<4,1\left(\sum_{\substack{r_{1} \bmod 6 \\ r_{2} \bmod 6}} \gamma_{\varepsilon}\left(r_{1}, r_{2}\right) \delta\left(r_{1}\right) \delta\left(r_{2}\right) M_{\varepsilon}\left(r_{1}, r_{2}\right)\right) P^{2}(\log P)^{4} .
$$

Démonstration. On reprend la majoration (2.1) établie dans la proposition 2.1 et on constate qu'il suffit de montrer que

$$
\sum_{\substack{0<k_{i} \leqslant P \\ 0<k_{1}+k_{2} \leqslant P \\ k_{i} \equiv r_{i}(\bmod 6)}} d_{3}\left(k_{1}\right) d_{3}\left(k_{2}\right)<\frac{4,1}{8} \delta\left(r_{1}\right) \delta\left(r_{2}\right) P^{2}(\log P)^{4},
$$

ce qui est une conséquence immédiate du lemme (3.4) en posant

$$
\begin{array}{ll}
a_{i}=\sum_{\substack{(i-1) P<40 k_{1} \leqslant i P \\
k_{1} \equiv r_{1}(\bmod 6)}} d_{3}\left(k_{1}\right), \quad a_{i}^{\prime}=\frac{\delta\left(r_{1}\right)}{40} P \log ^{2} P, \\
b_{i}=\sum_{\substack{(i-1) P<40 k_{2} \leqslant i P \\
k_{2} \equiv r_{2}(\bmod 6)}} d_{3}\left(k_{2}\right), & b_{i}^{\prime}=\frac{\delta\left(r_{2}\right)}{40} P \log ^{2} P .
\end{array}
$$

Le corollaire de la proposition 3.3 assure que les conditions du lemme sont satisfaites, et l'on a

$$
\begin{aligned}
\sum_{\substack{0<k_{i} \leqslant P \\
0<k_{i}+k_{2} \leqslant P \\
k_{i} \equiv r_{i}(\bmod 6)}} d_{3}\left(k_{1}\right) d_{3}\left(k_{2}\right) & <\sum_{i+j \leqslant 40} a_{i} b_{j} \sum_{i+j \leqslant 40} a_{i}^{\prime} b_{j}^{\prime} \\
& =\frac{40 \times 41}{2} \cdot \frac{\delta\left(r_{1}\right) \delta\left(r_{2}\right)}{40^{2}} P^{2} \log ^{4} P .
\end{aligned}
$$

\section{Majorations "racine-bornées".}

4a. Etude théorique. L'idée qui va être mise en oeuvre dans ce paragraphe est la suivante: étant donné une fonction arithmétique $f$, multiplicative et à valeurs positives, on cherche une fonction $g$, multiplicative et à valeurs 
positives également, et telle qu'il existe une constante $C$ pour laquelle la majoration

$$
f(n) \leqslant 2 C \sum_{\delta \leqslant \sqrt[n]{n}} g(\delta)
$$

soit vraie pour tout $n$.

Cette idée de sommer sur les diviseurs de $n$ avec une contrainte de taille se trouve chez van der Corput [3], puis chez Erdós [5] (limitation relative aux facteurs premiers des diviseurs) et a été reprise par McDonagh puis Wolke ([9]: limitation des diviseurs eux-mêmes par une puissance $n^{z}$ ), mais avec une mise en oeuvre tout à fait différente.

A la fonction $g$ on associe une transformée $G^{*}$ définie par

$$
G^{*}(n)=\sum_{\delta \mid n} \min (g(\delta), g(n / \delta))
$$

et l'on vérifie immédiatement que (si $g$ est multiplicative) $G^{*}$ est "surmultiplicative", i.e. que pour tous $m$ et $n$ premiers entre eux, on a $G^{*}(m n)$ $\geqslant G^{*}(m) G^{*}(n)$.

Comme on a trivialement $G^{*}(n) \leqslant 2 \sum_{\substack{\delta \mid n_{-} \\ \delta \leqslant, n}} g(\delta)$, toute majoration $f(n) \leqslant C G^{*}(n)$ implique automatiquement une majoration

$$
f(n) \leqslant 2 C \sum_{\substack{\delta ! n \\ \delta}} g(\delta),
$$

qui est précisément ce que l'on recherche. Le problème qui sera donc considéré maintenant est celui de la détermination du maximum d'une fonction "sous-multiplicative" (en l'occurence $f / G^{*}$ ), détermination dont on constatera qu'elle s'effectue conjointement avec la démonstration que la fonction considérée est bornée.

Pour les fonctions que nous aurons à considérer, les valeurs $f^{\prime}\left(p^{x}\right)$ et $g\left(p^{x}\right)$ ne dépendront que de $\alpha$, de sorte que les valeurs de $f(n)$, de $G^{*}(n)$, et de leur quotient, ne dépendront que de la suite des exposants $\left(\alpha_{1}, \alpha_{2}, \ldots, \alpha_{k}\right)$ dans la décomposition $n=p_{1}^{\alpha_{1}} p_{2}^{\alpha_{2}} \ldots p_{k}^{\alpha_{k}}$. Cela nous conduit à formaliser cette situation.

Définitions et notations. 1 . On appelle structure multiplicative $s$ une suite finie décroissante (au sens large) $\alpha_{1} \geqslant \alpha_{2} \geqslant \ldots \geqslant \alpha_{k} \geqslant 1$ d'entiers tous supérieurs ou égaux à 1 ; une structure multiplicative sera représentée indifféremment par la suite finie $\left(\alpha_{1}, \alpha_{2}, \ldots, \alpha_{k}\right)$ ou. la suite infinie $\left(\alpha_{1}, \alpha_{2}, \ldots, \alpha_{k}, 0,0, \ldots\right)$; si $n=p_{1}^{\alpha_{1}} p_{2}^{\alpha_{2}} \ldots p_{k}^{\alpha_{k}}$ et si, dans cette écriture les exposants sont ordonnés de façon décroissante, on dit que $s$ est la structure multiplicative de $n$.

2. On note $S$ l'ensemble de toutes les structures multiplicatives.
3. On dit qu'une fonction arithmétique $\varphi$ est structurale si ses valeurs sont déterminées par la structure multiplicative des entiers (bien entendu, $\varphi$ se factorise alors à travers $S$, et l'on notera de façon naturelle $\varphi(s)$ la valeur commune de $\varphi(n)$ pour les entiers $n$ de structure multiplicative $s)$.

4. Pour $h$ entier $\geqslant 1$, on définit la fonction $v_{h}$ par $v_{h}(s)=x_{h}$ le $h$-ième terme (en écriture infinie) de $s \in S$.

5. On appelle longueur de $s \in S$ et on note $l(s)$ le nombre de termes non nuls de $s$ (on a donc $\alpha_{l(s)} \geqslant 1$ et $\alpha_{l(s)+1}=0$ ).

6. Si $s=\left(\alpha_{1}, \ldots, \alpha_{l(s)}\right)$ et $t=\left(\beta_{1}, \ldots, \beta_{l(t)}\right)$, et si $\alpha_{l(s)} \geqslant \beta_{1}$, on note $s+t$ la structure multiplicative concaténée $\left(\alpha_{1}, \ldots, \alpha_{l(s)}, \beta_{1}, \ldots, \beta_{u(t)}\right)$.

7. On note $S(s)$ l'ensemble des structures multiplicatives qui commencent par $s, y$ compris $s$ elle-même.

On peut ordonner naturellement $S$ par l'ordre lexicographique, et lon explicite ci-dessous quelques remarques ou propriétés relatives à cet ordre, que l'on note $\leqslant$ :

- $\leqslant$ est un bon ordre total;

- si $s=\left(\alpha_{1}, \ldots, \alpha_{l}\right)$, avec $\alpha_{1}>x_{2}>\ldots>\alpha_{k}=\alpha_{k+1}=\ldots=\alpha_{1}$, et si l'on note $\sigma(s)$ le minimum de $\left.\left\{s^{\prime}\right\} s^{\prime}>S(s)\right\}$, alors on a $\sigma(s)=\left(\alpha_{1}, \ldots, x_{k-1}\right.$, $\left.\alpha_{k}+1\right)$

- $\forall s \forall s^{\prime} s^{\prime} \geqslant s \Rightarrow s^{\prime} \in S(s)$ ou $s^{\prime} \geqslant \sigma(s)$.

Proposition 4.1. Soit $\varphi$ une fonction arithmétique structurale, sousmultiplicative et à valeurs positives. Pour $s \in S$, on pose $C(s):=\max \{\varphi(t) \mid t$ $<s_{\}}^{\}}$. Alors on a, pour tout $s$, les relations:

si $\varphi(s)>1$,

$$
C(s+1)=\max (C(s), \varphi(s))
$$

si $\varphi(s) \leqslant 1$

$$
C(\sigma(s))=C(s)
$$

Corollatre. Soit un entier $\alpha$. Si l'on sait, primo (en faisant fonctionner l'alyorithme qui découle de l'énoncé de la proposition précédente), que C $((\alpha))$ $<\infty$, secundo (par une preuve théorique), que pour $\beta \geqslant \alpha$ on a $\varphi((\beta)) \leqslant 1$, alors la fonction $\varphi$ est bornée et l'on a

$$
\max \{\varphi(s) \mid s \in S\}=C((x)) \text {. }
$$

Démonstration de la proposition 4.1. Si $C(s)=+\infty$ il n'y a rien à démontrer.

Si $C(s)<+\infty$ et $\varphi(s)>1$ la propriété est triviale puisque $s+1$ est le successeur de $s$.

Si $C(s)<+\infty$ et $\varphi(s) \leqslant 1$ il faut considérer les éléments $s^{\prime} \in S$, supérieurs ou égaux à $s$, mais strictement inférieurs à $\sigma(s)$. De tels éléments appartiennent à $S(s)$ et peuvent donc s'écrire $s^{\prime}=s+s^{\prime \prime}$ avec $v_{1}\left(s^{\prime \prime}\right) \leqslant v_{l(s)} \leqslant v_{1}(s)$. Deux éventualités sont alors possibles: 
linégalité

- ou bien $s^{\prime \prime}<s$ (cas général), et la sous-multiplicativité de $\varphi$ implique

$$
\varphi\left(s^{\prime}\right) \leqslant \varphi(s) \varphi\left(s^{\prime \prime}\right) \leqslant \varphi\left(s^{\prime \prime}\right) \leqslant C(s)
$$

- ou bien $s^{\prime \prime}=s+\ldots+s+s^{\prime \prime \prime}$ avec $s^{\prime \prime \prime}<s$ (cas particulier $s=(\alpha, \ldots, \alpha)$ et $\left.s^{\prime \prime}=(\alpha, \ldots, \alpha, \ldots)\right)$, et la sous-multiplicativité implique de même

$$
\varphi\left(s^{\prime}\right) \leqslant(\varphi(s))^{m} \varphi\left(s^{\prime \prime \prime}\right) \leqslant \varphi\left(s^{\prime \prime}\right) \leqslant C(s) .
$$

Démonstration du lemme. Comme pour tout $s \in S$ il existe un entier $\beta$ tel que $s<(\beta)$, il suffit de montrer que, pour tout $\beta \geqslant \alpha$, on a

$$
C((\beta)) \leqslant C((\alpha)) \text {. }
$$

Cela s'effectue par récurrence car, d'après la proposition 4.1 , on a

$$
[C((\beta))=C((\alpha)) \text { et } \varphi((\beta)) \leqslant 1] \Rightarrow C((\beta+1))=C(\sigma((\beta))) \leqslant C((\alpha)) \text {. }
$$

L'algorithme de calcul de $C((\alpha))$ qui découle de la proposition 4.1 est le suivant:

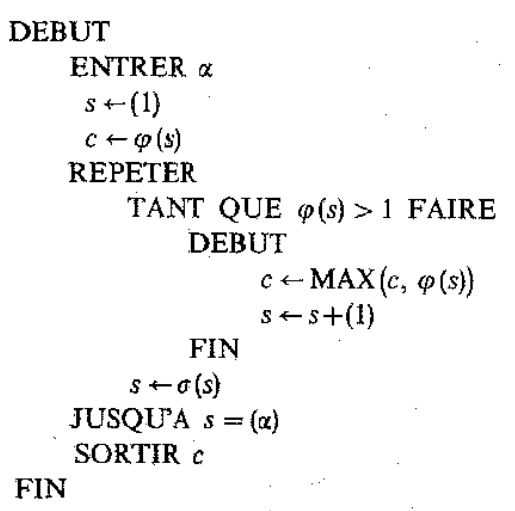

La programmation de cet algorithme ne soulève pas d'autre difficulté que la construction d'une structure de données efficace pour manipuler les structures multiplicatives.

\section{4b. Application à la fonction $d_{3}$.}

Proposition 4.2. Soient $f$ la fonction de diviseurs $d_{3}$ et $g$ la fonction multiplicative définie sur les puissances des nombres premiers par

$$
\begin{aligned}
g(p) & =2,5 \\
g\left(p^{x}\right) & =2,5 \alpha-0,5 \quad \text { pour } \quad \alpha \geqslant 2 .
\end{aligned}
$$

Alors on a pour tout $n$

$$
f(n) \leqslant 2 C \sum_{\substack{\delta \mid n_{\sqrt[n]{n}} \\ \delta \leqslant \sqrt{n}}} g(\delta),
$$

avec $2 C=243 / 76=3,19736 \ldots$

La démonstration est présentée avec les notations du sous-paragraphe précédent: $G^{*}$ et $\varphi=f / G^{*}$.

La première moitié de la démonstration est le résultat "théorique" suivant: pour $\beta \geqslant 15$, on a $\varphi((\beta)) \leqslant 1$.

Si $\beta=2 \beta^{\prime}$, on a

$$
f((\beta))=\left(2 \beta^{\prime}+1\right)\left(2 \beta^{\prime}+2\right) / 2=2 \beta^{\prime 2}+3 \beta^{\prime}+1,
$$

$$
\begin{aligned}
G^{*}((\beta)) & =2\left(1+2,5+4,5+\ldots+2,5\left(\beta^{\prime}-1\right)-0,5\right)+2,5 \beta^{\prime}-0,5 \\
& =2,5 \beta^{\prime 2}-\beta^{\prime}+3,5,
\end{aligned}
$$

et alors $f((\beta)) / G^{*}((\beta))<1$ dès que $\beta \geqslant 16$.

Si $\beta=2 \beta^{\prime}+1$, on a

$$
f((\beta))=\left(2 \beta^{\prime}+2\right)\left(2 \beta^{\prime}+3\right) / 2=2 \beta^{\prime 2}+5 \beta^{\prime}+3,
$$

$$
G^{*}((\beta))=2\left(1+2,5+4,5+\ldots+2,5 \beta^{\prime}-0,5\right)=2,5 \beta^{2}+1,5 \beta^{\prime}+3,
$$

et alors $f((\beta)) / G^{*}((\beta)) \leqslant 1$ dès que $\beta \geqslant 15$.

La deuxième moitié de la démonstration consiste à calculer $C((15))$ en utilisant l'algorithme indiqué précédemment. Il n'y a aucune difficulté supplémentaire autre que la nécessité d'une programmation efficace de la procédure de calcul de $G^{*}(s)$, et l'on trouve $C((15))=243 / 152=1,598 \ldots$. (valeur atteinte pour $s=(1,1,1,1,1)$ ).

5. Nombre de solutions de congruences quadratiques. Il faut maintenant transformer l'expression du majorant $M_{\varepsilon}\left(r_{1}, r_{2}\right)$ en utilisant les résultats du paragraphe précédent. Commençons par introduire la fonction $\varrho_{\varepsilon}\left(h_{1}, h_{2} ; \delta\right)$, nombre de solutions en $y$ modulo $\delta$ de la congruence

$$
3\left(2 y+\varepsilon+h_{1}+h_{2}\right)^{2}+h_{1}^{2}+h_{2}^{2} \equiv 0(\bmod \delta),
$$

fonction qui est trivialement multiplicative en $\delta$.

On a alors

$$
\begin{aligned}
& M_{\varepsilon}\left(r_{1}, r_{2}\right)=\max _{\substack{h_{1} h_{2} \neq 0 \\
\left|h_{1}\right|+\left|h_{2}\right| \leqslant P}}\left(\sum_{\substack{2 P<2 y+\varepsilon \leqslant 4 P \\
2 P<2 y+\varepsilon+h_{1}+h_{2} \leqslant 4 P}} d_{3}\left(3\left(2 y+\varepsilon+h_{1}+h_{2}\right)^{2}+h_{1}^{2}+h_{2}^{2}\right)\right) \\
& h_{i} \equiv r_{i}(\bmod 6) \\
& \leqslant \max _{\substack{h_{1} h_{2} \neq 0 \\
\left|h_{1}\right|+\left|h_{2}\right| \leqslant P \\
h_{i}=r_{i}(\bmod 6)}}\left(2 C \sum_{\substack{2 P<2 y+\varepsilon \leqslant 4 P \\
2 P<2 y+\varepsilon+h_{1}+h_{2} \leqslant 4 P}} \sum_{\substack{\delta \mid n=3\left(2 y+\varepsilon+h_{1}+h_{2}\right)^{2}+h_{1}^{2}+h_{2}^{2} \\
\delta \leqslant \sqrt{n}}}\right.
\end{aligned}
$$


On note que les conditions sur $y, h_{1}$ et $h_{2}$, impliquent les inégalités $\left|2 y+\varepsilon+h_{1}+h_{2}\right| \leqslant 4 P$ et $h_{1}^{2}+h_{2}^{2} \leqslant P^{2}$, de sorte que la condition

$$
\delta \leqslant \sqrt{3\left(2 y+\varepsilon+h_{1}+h_{2}\right)^{2}+h_{1}^{2}+h_{2}^{2}}
$$

implique l'inégalité $\delta \leqslant 7 P$. On peut alors permuter les sommations et écrire

$$
\begin{aligned}
M_{\varepsilon}\left(r_{1}, r_{2}\right) & \leqslant 2 C \max _{\substack{h_{1} h_{2} \neq 0 \\
\left|h_{1}\right|+h_{2} \mid \leqslant P \\
h_{i}=r_{i}(\operatorname{mul} \sigma)}}\left(\sum_{\delta \leqslant 7 P} g(\delta) \sum_{\substack{2 P<2 y+\varepsilon \leqslant 4 P \\
\delta \mid 3\left(2 y+\varepsilon+h_{1}+h_{2}\right)^{2}+h_{1}^{2}+h_{2}^{2}}} 1\right) \\
& \leqslant 2 C \max _{\substack{h_{i} \equiv r_{i}(\bmod 6) \\
\sum}} \sum_{\delta \leqslant 7 P} g(\delta) \varrho_{\varepsilon}\left(h_{1}, h_{2} ; \delta\right)([P / \delta]+1) \\
& \leqslant 14 C\left(\max _{\substack{h_{i} \equiv r_{i}(\bmod 6) \\
\sum}} \frac{g(\delta) \varrho_{\varepsilon}\left(h_{1}, h_{2} ; \delta\right)}{\delta}\right) P .
\end{aligned}
$$

$$
\text { qu’à majorer les sommes }
$$

$$
S=\sum_{\delta \leqslant 7 P} \frac{g(\delta) \varrho_{\varepsilon}\left(h_{1}, h_{2} ; \delta\right)}{\delta}
$$

ce qui sera fait au paragraphe 6 et dernier (Nota: La majoration de $[P / \delta]$ +1 par $7 P / \delta$ est motivée par le fait que les techniques qui seront employées ne permettraient pas de majorer $\sum_{\delta \leqslant 7 P} g(\delta) \varrho_{\varepsilon}\left(h_{1}, h_{2} ; \delta\right)$ par mieux que 7PS). Auparavant, il faut estimer les fonctions $\varrho_{\varepsilon}\left(h_{1}, h_{2} ; \delta\right)$ pour les puissances des nombres premiers.

Proposition 5.1. Selon les cas détaillés ci-dessous, la suite $\alpha \mapsto \varrho_{\varepsilon}\left(h_{1}, h_{2} ; p^{\alpha}\right)$ est majorée terme à terme (pour $\alpha=1,2, \ldots$ ) par:
si $p \geqslant 5$
$2,2, \quad 2,2, \quad 2,2, \ldots$
si $\quad p^{2} \nmid h_{1}^{2}+h_{2}^{2}$
$1, p, 2 p, p^{2}, 2 p^{2}, p^{3}, 2 p^{3}, p^{4}, \ldots$
si $p^{2} \mid h_{1}^{2}+h_{2}^{2}$;
si $p=3$
$0,0,0,0,0,0, \ldots$
si $3 \nmid h_{1}^{2}+h_{2}^{2}$,
$3,3,9,18,27,54,81,162, \ldots$
si $3 \mid h_{1}^{2}+h_{2}^{2}$
si $p=2$
$0,0,0,0,0,0, \ldots$
si $\varepsilon=1$,
$2,0,0,0,0,0, \ldots$
si $\varepsilon=0$ et $2 \nmid h_{1} h_{2}$,
$2,4,8,8,8,8,8,8, \ldots$
si $\varepsilon=0$ et $2 \nmid h_{1}$ et $2 \mid h_{2}$ (ou inversement),
$2,4,4,8,16,16,32,32,64, \ldots$
si $\varepsilon=0$ et $2 \mid h_{1}$ et $2 \mid h_{2}$.

Démonstration pour $p \geqslant 5$. Si $p^{2} \nmid h_{1}^{2}+h_{2}^{2}$ mais $p \mid h_{1}^{2}+h_{2}^{2}$, la suite des $\varrho_{\varepsilon}\left(h_{1}, h_{2} ; p^{\alpha}\right)$ est trivialement $1,0,0,0, \ldots$

Si $p \nmid h_{1}^{2}+h_{2}^{2}$, la congruence (5.1) pour $\delta=p$ possède 0 ou 2 solutions et, "au-dessus" de chaque solution pour $p^{\alpha}$, il y a exactement une solution pour $p^{\alpha+1}$. Soit en effet $u$ une solution de (5.1) pour $p$, et cherchons sous la forme $y+t p^{\alpha}$ ( $t$ défini modulo $p$ ) une solution pour $p^{\alpha+1}$. On a la congruence

$$
\begin{aligned}
3\left(2\left(y+t p^{\alpha}\right)+\varepsilon+h_{1}+h_{2}\right)^{2} & \\
& \equiv 3\left(2 y+\varepsilon+h_{1}+h_{2}\right)^{2}+36 t\left(2 y+\varepsilon+h_{1}+h_{2}\right) p^{\alpha}\left(\bmod p^{\alpha+1}\right) .
\end{aligned}
$$

Mais $y$ est solution de (5.1) pour $p^{\alpha}$ et $p$ ne divise pas $h_{1}^{2}+h_{2}^{2}$, donc $p$ ne divise pas non plus $36\left(2 y+\varepsilon+h_{1}+h_{2}\right)$, et il existe par conséquent une unique valeur de $t$ convenable.

Dans les deux cas la suite des $\varrho_{\varepsilon}\left(h_{1}, h_{2} ; p^{x}\right)$ est bien majorée par $2,2,2,2, \ldots$

Si maintenant $p^{2} \mid h_{1}^{2}+h_{2}^{2}$, définissons $\beta$ par $p^{\beta} \mid h_{1}^{2}+h_{2}^{2}$. On doit alors distinguer trois cas:

- pour $\alpha \leqslant \beta$, la congruence (5.1) se réduit à

$$
3\left(2 y+\varepsilon+h_{1}+h_{2}\right)^{2} \equiv 0\left(\bmod p^{\alpha}\right)
$$

et donc à

$$
3\left(2\left(z+u 2^{\alpha-2}\right)+1\right)^{2} \equiv 3(2 z+1)^{2}+3 u(2 z+1) 2^{\alpha}\left(\bmod 2^{\alpha+1}\right),
$$

il y a donc $p^{\alpha-[(\alpha+1) / 2]}=p^{[\alpha / 2]}$ solutions en $y$ modulo $p^{\alpha}$, et une suite majorante est alors $1, p, p, p^{2}, p^{2}, p^{3}, \ldots$;

- pour $\alpha>\beta$ et $\beta$ impair, il n'y a trivialement aucune solution;

- pour $\alpha>\beta$ et $\beta$ pair, enfin, le fait que y soit solution de (5.1) pour $p^{\alpha}$ implique que $p^{\beta / 2}$ divise $2 y+\varepsilon+h_{1}+h_{2}$; on pose donc $2 y+\varepsilon+h_{1}+h_{2}=z p^{\beta / 2}$ ( $z$ défini modulo $p^{\alpha-(\beta / 2)}$ ), on pose $h_{1}^{2}+h_{2}^{2}=a p^{\beta}$, avec donc $p \nmid a$, et l'on doit maintenant résoudre la congruence

$$
3 z^{2}+a \equiv 0\left(\bmod p^{\alpha-\beta}\right)
$$

qui possède (cf. le début de cette démonstration) au plus deux solutions en $z$ modulo $p^{\alpha-\beta}$ et donc au plus $2 p^{\beta / 2}$ solutions en $z$ modulo $p^{\alpha-\beta / 2}$; ce qui donne enfin au plus $2 p^{\beta / 2}$ solutions en $y$ modulo $p^{\alpha}$, et une suite majorante est alors $1, p, p, p^{2}, \ldots, p^{\beta / 2}, 2 p^{\beta / 2}, 2 p^{\beta / 2}, \ldots$, elle même majorée par $1, p, 2 p$, $p^{2}, \ldots, p^{\beta / 2}, 2 p^{\beta / 2}, p^{(\beta / 2)+1}, \ldots$

Démonstration pour $p=3$. Si $3 \Varangle h_{1}^{2}+h_{2}^{2}$, la congruence (5.1) est trivialement impossible.

Si $3 \mid h_{1}^{2}+h_{2}^{2}$ et $\alpha=1$ (i.e. $\delta=3$ ), la congruence (5.1) possède trois solutions.

Si $3 \mid h_{1}^{2}+h_{2}^{2}$ et $\alpha>1$, le nombre de solutions en $y$ (modulo $3^{\alpha}$ ) de la congruence (5.1) est trois fois le nombre de solutions en $y$ (modulo $3^{\alpha-1}$ ) de 
la congruence

$$
\left(2 y+\varepsilon+h_{1}+h_{2}\right)^{2}+\left(h_{1}^{2}+h_{2}^{2}\right) / 3 \equiv 0\left(\bmod 3^{\alpha-1}\right)
$$

pour laquelle on peut répéter très exactement la démonstration du cas $p \geqslant 5$, et on obtient ainsi que la suite $\varrho_{z}\left(h_{1}, h_{2} ; 3\right)$ est majorée par la suite 3 , $3\left(1,3,2 \cdot 3,3^{2}, 2 \cdot 3^{2}, \ldots\right)=3,3,3^{2}, 2 \cdot 3^{2}, \ldots$

Démonstration pour $p=2$. Si $\varepsilon=1,3\left(2 y+\varepsilon+h_{1}+h_{2}\right)^{2}$ et $h_{1}^{2}+h_{2}^{2}$ sont de parités différentes, et la congruence (5.1) est donc impossible.

Si $\varepsilon=0$ et $2 \Varangle h_{1} h_{2}$ (i.e. $h_{1}$ et $h_{2}$ tous deux impairs), il faut distinguer entre $\alpha=1$ et $\alpha>1$. Pour $\alpha=1$ (i.e. $\delta=2$ ), la congruence (5.1) possède trivialement deux solutions. Pour $\alpha>1,3\left(2 y+h_{1}+h_{2}\right)^{2} \equiv 0(\bmod 4)$ et $h_{1}^{2}$ $+h_{2}^{2} \equiv 2(\bmod 4)$, et la congruence $(5.1)$ est donc impossible.

Si $\varepsilon=0$ et $2 \nmid h_{1}$ et $2 \mid h_{2}$ (ou inversement), on pose $z=y+\frac{1}{2}\left(h_{1}+h_{2}-1\right.$ ) et $K=-h_{1}^{2}-h_{2}^{2}$, ce qui réduit la congruence (5.1) à la forme

$$
3(2 z+1)^{2} \equiv K\left(\bmod 2^{\alpha}\right) .
$$

On constate alors que l'on a, pour $\alpha \geqslant 3$, les deux congruences:

$$
\begin{aligned}
& 3\left(2\left(z+t 2^{\alpha-2}\right)+1\right)^{2} \equiv 3(2 z+1)^{2}\left(\bmod 2^{\alpha}\right) \\
& 3\left(2\left(z+u 2^{\alpha-2}\right)+1\right)^{2} \equiv 3(2 z+1)^{2}+3 u(2 z+1) 2^{\alpha}\left(\bmod 2^{\alpha+1}\right)
\end{aligned}
$$

ce qui prouve deux choses:

- primo que les solutions de (5.3) peuvent être groupées par paquets de quatre $(t=0,1,2$, ou 3$)$;

- secundo qu'au-dessus de chaque paquet de quatre solutions de (5.3) pour $2^{\alpha}$, il existe exactement un paquet de quatre solutions de (5.3) pour $2^{\alpha+1}$ (correspondant à $u=0,2,4$, ou 6 , ou bien à $u=1,3,5$, ou 7 ).

Il suffit maintenant de compter les solutions jusqu'à $2^{\alpha}=8$, soit:

2 solutions pour $2^{\alpha}=2$,

4 solutions pour $2^{\alpha}=4$,

8 solutions pour $2^{\alpha}=8$ et $h_{2} \equiv 2(\bmod 4)$,

pas de solution pour $2^{\alpha}=8$ et $h_{2} \equiv 0(\bmod 4)$,

et cela montre la majoration des $\varrho_{0}\left(h_{1}, h_{2} ; 2^{\alpha}\right)$ par la suite $2,4,8,8,8,8, \ldots$ dans tous les cas.

Dernière éventualité enfin, si $\varepsilon=0,2 \mid h_{1}$ et $2 \mid h_{2}$. On réduit la congruence $(5.1)$ de la façon suivante: on définit $\beta(\geqslant 2)$ par $2^{\beta} \| h_{1}^{2}+h_{2}^{2}$ et on pose $\beta^{\prime}=[\beta / 2]$; on constate alors que $2^{\beta^{\prime}} \mid h_{1}$ et $2^{\beta^{\prime}} \mid h_{2}$, et que l'un au moins des deux quotients $h_{i} / 2^{\beta^{*}}$ est impair. On peut donc réécrire la congruence (5.1)

$$
3\left(2 y+h_{1}+h_{2}\right)^{2}+2^{2 \beta^{\prime}}\left\{\left(\frac{h_{1}}{2^{\beta^{\prime}}}\right)^{2}+\left(\frac{h_{2}}{2^{\beta^{\prime}}}\right)^{2}\right\} \equiv 0\left(\bmod 2^{\alpha}\right)
$$

et deux cas sont alors à distinguer:
- pour $\alpha \leqslant 2 \beta^{\prime}$, la congruence se réduit à

$$
3\left(2 y+h_{1}+h_{2}\right)^{2} \equiv 0\left(\bmod 2^{x}\right)
$$

et donc à

$$
2 y+h_{1}+h_{2} \equiv 2 y \equiv 0\left(\bmod 2^{(i x+1) / 2\}}\right),
$$

laquelle possède deux solutions en $y$ modulo $2^{[(x+1) / 2]}$, et donc $2^{x-[(x+1) / 2]+1}$ $=2^{[\alpha / 2]+1}$ solutions en $y$ modulo $2^{x}$;

- pour $\alpha>2 \beta^{\prime}, 2^{\beta^{\prime}}$ divise nécessairement $2 y+h_{1}+h_{2}$, de sorte que lon peut poser $y=2^{\beta^{\prime}-1} z$, et la congruence se réduit à

$$
3\left(z+\frac{h_{1}}{2^{\beta^{\prime}}}+\frac{h_{2}}{2^{\beta^{\prime}}}\right)^{2}+\left(\frac{h_{1}}{2^{\beta^{\prime}}}\right)^{2}+\left(\frac{h_{2}}{2^{\beta^{\prime}}}\right)^{2} \equiv 0\left(\bmod 2^{\alpha-2 \beta^{\prime}}\right) ;
$$

si $z$ est impair, cette congruence est impossible (cf. le début de cette étude, cas $\varepsilon=1$ ); $z$ est donc pair, soit $z=2 z^{\prime}$, et le nombre de solutions de (5.4) en $z^{\prime}$ modulo $2^{\alpha-2 \beta^{\prime}}$ est donc majoré par 2,4 , ou 8 , selon que $\alpha-2 \beta^{\prime}$ est égal à 1 , à 2, ou supérieur ou égal à 3 (cf. la partie précédente de cette étude, cas $\varepsilon=0$ et $h_{1}$ ou $h_{2}$ impair); cela donne donc les majorations suivantes:

1,2 , ou 4 , pour les solutions en $z$ modulo $2^{\alpha-2 \beta^{\prime}}$,

1,2 , ou 4 , pour les solutions en $y$ modulo $2^{\alpha-\beta^{\prime}-1}$,

$2^{\beta^{\prime}+1}, 2^{\beta^{\prime}+2}$, ou $2^{\beta^{\prime}+3}$, pour les solutions en $y$ modulo $2^{\alpha}$.

Si on récapitule les résultats obtenus par les deux cas, $\alpha \leqslant 2 \beta^{\prime}$, puis $\alpha>2 \beta^{\prime}$, on obtient:

\begin{tabular}{lllllllllllllll}
\hline$\alpha$ & 1 & 2 & 4 & $\ldots$ & $2 \beta^{\prime}-1$ & $2 \beta^{\prime}$ & $2 \beta^{\prime}+1$ & $2 \beta^{\prime}+2$ & $2 \beta^{\prime}+3$ & $2 \beta^{\prime}+4$ & $2 \beta^{\prime}+5$ & $\ldots$ \\
\hline $\begin{array}{l}\text { exposant } \\
\text { de la } \\
\text { majoration }\end{array}$ & 1 & 2 & 2 & 3 & $\ldots$ & $\beta^{\prime}$ & $\beta^{\prime}+1$ & $\beta^{\prime}+1$ & $\beta^{\prime}+2$ & $\beta^{\prime}+3$ & $\beta^{\prime}+3$ & $\beta^{\prime}+3$ & $\ldots$ \\
\hline
\end{tabular}

et la suite des majorations est bien, pour toutes les valeurs de $\beta^{\prime}$, majorée par la suite $2,4,4,8,16,16,32,32,64, \ldots$

\section{Majorations finales}

6a. Majoration de $M_{\varepsilon}\left(r_{1}, r_{2}\right)$. Il s'agit (cf. formule (5.2)) de majorer la somme

$$
\sigma_{\varepsilon}\left(h_{1}, h_{2} ; P\right)=\sum_{\delta \leqslant 7 P} \frac{g(\delta) \varrho_{2}\left(h_{1}, h_{2} ; \delta\right)}{\delta},
$$

ce qui s'effectuera grâce à trois "outils":

- la séparation des facteurs locaux relatifs à $p=2$ et $p=3$; 
- la majoration par une somme similaire portant sur une fonction simple:

-. le fait que les fonctions ainsi introduites soient liées par des produits de comvolution àdes fonctions très faciles àsommer

Dans tout ce qui suit, on marquera d'un «'» tout ce qui concerne le "facteur de convolution" relatif à $p=2$ et $p=3$, et d'un " "» tout ce qui concerne le "facteur de convolution" relatif aux $p \geqslant 5$. Ainsi, si $f$ est une fonction arithmétique multiplicative, on a

$$
\begin{aligned}
& f^{\prime}(n)= \begin{cases}f(n) & \text { si } n=2^{a} 3^{b} \\
0 & \text { sinon; }\end{cases} \\
& f^{\prime \prime}(n)= \begin{cases}f(n) & \text { si }(n, 6)=1 \\
0 & \text { sinon }\end{cases}
\end{aligned}
$$

(on a la relation $f=f^{\prime} * f^{\prime \prime}$ ). On utilisera notamment les deux fonctions sommatoires

$$
\sigma_{\varepsilon}^{\prime \prime}\left(h_{1}, h_{2} ; P\right)=\sum_{\substack{\delta \leqslant 7 P \\ \delta, 6)=1}} \frac{g(\delta) \varrho_{\varepsilon}\left(h_{1}, h_{2} ; \delta\right)}{\delta}
$$

et

$$
S_{\varepsilon}^{\prime \prime}\left(h_{1}, h_{2} ; P\right)=\sum_{\substack{\delta \leqslant 7 P \\(\delta, 6)=1}} g(\delta) \varrho_{\varepsilon}\left(h_{1}, h_{2} ; \delta\right)
$$

Proposition 6.1. On pose:

\begin{tabular}{|c|c|c|c|c|}
\hline \multicolumn{5}{|c|}{$\mu_{0}\left(r_{1}, r_{2}\right)$} \\
\hline$r_{1}$ & 0 & 1 ou 5 & 2 ou 4 & 3 \\
\hline 0 & $m_{4} m_{3}$ & $m_{2}$ & $m_{4}$ & $m_{2} m_{3}$ \\
\hline 1 ou 5 & $m_{2}$ & $m_{0}$ & $m_{2}$ & $m_{0}$ \\
\hline 2 ou 4 & $m_{4}$ & $m_{2}$ & $m_{4}$ & $m_{2}$ \\
\hline 3 & $m_{2} m_{3}$ & $m_{0}$ & $m_{2}$ & $m_{0} m_{3}$ \\
\hline
\end{tabular}

$$
\begin{aligned}
& m_{0}=1+\frac{2,5 \times 2}{2}=3,5 \\
& m_{2}=1+\frac{2,5 \times 2}{2}+\frac{4,5 \times 4}{4}+\frac{7 \times 8}{8}+\frac{9,5 \times 8}{16}+\ldots=27 \\
& m_{4}=1+\frac{2,5 \times 2}{2}+\frac{4,5 \times 4}{4}+\frac{7 \times 4}{8}+\frac{9,5 \times 8}{16} \\
& \quad+\frac{12 \times 16}{32}+\frac{14,5 \times 16}{64}+\frac{17 \times 32}{128}+\ldots=43 \\
& m_{3}=1+\frac{2,5 \times 3}{3}+\frac{4,5 \times 3}{9}+\frac{7 \times 9}{27}+\frac{9,5 \times 18}{81} \\
& \quad+\frac{12 \times 27}{243}+\frac{14,5 \times 54}{729}+\frac{17 \times 81}{2187}+\ldots=13,75
\end{aligned}
$$

\begin{tabular}{|c|c|c|c|c|}
\hline \multicolumn{5}{|c|}{$\mu_{1}\left(r_{1}, r_{2}\right)$} \\
\hline$r_{1}$ & 0 & 1 ou 5 & 2 ou 4 & 3 \\
\hline 0 & $m_{3}$ & 1 & 1 & $m_{3}$ \\
\hline 1 ou 5 & 1 & 1 & 1 & 1 \\
\hline 2 ou 4 & 1 & 1 & 1 & 1. \\
\hline 3 & $m_{3}$ & 1 & 1 & $m_{3}$ \\
\hline
\end{tabular}

On définit les coefficients numériques $\mu_{\varepsilon}\left(r_{1}, r_{2}\right)$ (fonctions des classes $r_{\check{i}}$ des $h_{i}$ mod 6) par les tableaux suivants:

Alors on a les majorations

$$
\sigma_{\varepsilon}\left(h_{1}, h_{2} ; P\right) \leqslant \mu_{\varepsilon}\left(r_{1}, r_{2}\right) \sigma_{\varepsilon}^{\prime \prime}\left(h_{1}, h_{2} ; P\right) .
$$

Démonstration. On considère la propriété (déjà énoncée et utilisée au paragraphe $3 \mathrm{~b}$ ), valable pour toute fonction multiplicative $h$ àvaleurs réelles positives,

$$
\begin{aligned}
\sum_{n \leqslant X} h(n) & \leqslant \sum_{m=2^{\alpha} 3^{\beta}} h(m) \sum_{\substack{n \leqslant X \\
(n, 6)=1}} h(n) \\
& =\left(\sum_{k=0}^{\infty} h\left(2^{k}\right)\right)\left(\sum_{k=0}^{\infty} h\left(3^{k}\right)\right) \sum_{\substack{n \leqslant X \\
(n, 6)=1}} h(n) .
\end{aligned}
$$

Les coefficients $m_{0}, m_{2}, m_{4}$, et $m_{3}$ sont précisément les valeurs des sommes

$$
\sum_{k=0}^{\infty} \frac{g\left(2^{k}\right) \varrho_{\varepsilon}\left(h_{1}, h_{2} ; 2^{k}\right)}{2^{k}} \quad \text { et } \quad \sum_{k=0}^{\infty} \frac{g\left(3^{k}\right) \varrho_{\varepsilon}\left(h_{1}, h_{2} ; 3^{k}\right)}{3^{k}}
$$

pour les valeurs non triviales des suites qui majorent dans la proposition 5.1 les suites $\varrho_{\varepsilon}\left(h_{1}, h_{2} ; 2^{k}\right)$ et $\varrho_{\varepsilon}\left(h_{1}, h_{2} ; 3^{k}\right)$. Les coefficients $\mu_{\varepsilon}\left(r_{1}, r_{2}\right)$ apparaissent 
alors comme des majorants de la fonction sommatoire locale $\sigma_{e}^{\prime}\left(h_{1}, h_{2} ; \infty\right)$ Ils sont fabriqués en utilisant, conformément à l'énoncé de la proposition 5.1, $m_{0}$ si $\varepsilon=0, h_{1}$ et $h_{2}$ impairs, $m_{2}$ si $\varepsilon=0, h_{i}$ pair et $h_{3-i}$ impair, $m_{4}$ si $\varepsilon=0$, $h_{1}$ et $h_{2}$ pairs, et enfin $m_{3}$ si $h_{1}$ et $h_{2}$ divisibles par 3 .

On va maintenant remplacer $g(\delta) \varrho_{\varepsilon}\left(h_{1}, h_{2} ; \delta\right) / \delta$ par une fonction plus simple, mais il faut auparavant donner l'inégalité de Deshouillers-DressHarlipó pour les fonctions arithmétiques, qui permettra d'obtenir l'inégalité adéquate entre les fonctions sommatoires.

LEMME 6.2. Soient $f$ et $g$ deux fonctions arithmétiques multiplicatives, à valeurs réelles positives, qui vérifient les inégalités

$$
\forall p \text { premier } \forall \alpha \in N \quad \sum_{k=0}^{\alpha} f\left(p^{k}\right) \leqslant \sum_{k=0}^{\alpha} g\left(p^{k}\right) \text {. }
$$

Alors on a, pour tout $X$, Tinégalité

$$
\sum_{n \leqslant X} f(n) \leqslant \sum_{n \leqslant X} g(n)
$$

Remarque. Les conditions sont optimales, comme on peut le constater en considérant deux fonctions $f$ et $g$ nulles sauf sur les puissances d'un nombre premier fixé.

Démonstration. On démontre le lemme dans le cas particulier où, $p$ étant un nombre premier fixé, on a $f(m)=g(m)$ si $(m, p)=1$. La conclusion découle alors trivialement de l'écriture

$$
\sum_{n \leqslant X} f(n)=\sum_{\substack{m \leqslant X \\(m, p)=1}} f(m)\left(\sum_{m p^{k} \leqslant X} f\left(p^{k}\right)\right)
$$

(et de l'écriture analogue de $\sum_{n \leqslant X} g(n)$ ).

Dans le cas général, cela permet de faire la démonstration "de proche en proche" (pour $X$ fixé, il n'y a qu'un nombre fini de nombres premiers à considérer)

Proposmron 6.3. Les entiers $h_{1}$ et $h_{2}$ étant fixés, on définit la fonction arithmétique multiplicative $g_{0}^{\prime \prime}(n)=g_{0}^{\prime \prime}\left(h_{1}, h_{2} ; n\right)$ de la façon suivante

si $p=2$ ou 3 et $\alpha \geqslant 1$ :

$$
g_{0}^{\prime \prime}\left(p^{\alpha}\right)=0
$$

si $p \geqslant 5, p \nmid h_{1}^{2}+h_{2}^{2}$ et $\alpha \geqslant 1$ :

$$
g_{0}^{\prime \prime}\left(p^{x}\right)=0
$$

si $p \geqslant 5, p \mid h_{1}^{2}+h_{2}^{2}$

$$
\begin{aligned}
g_{0}^{\prime \prime}(p) & =2, \\
g_{0}^{\prime \prime}\left(p^{x}\right) & =0 \quad \text { pour } \alpha \geqslant 2,
\end{aligned}
$$

puis on définit $g_{i}^{\prime \prime}(n)=g_{i}^{\prime \prime}\left(h_{1}, h_{2} ; n\right)(i=1,2, \ldots, 5)$ par $g_{i}^{\prime \prime}=g_{i-1}^{\prime \prime} * i^{\prime \prime}$, où $i^{\prime \prime}$ est la fonction arithmétique caractéristique des entiers premiers avec 6.

Alors on a, pour tout $X$, l'inégalité

$$
\sum_{\substack{n \leqslant X \\(n, 6)=1}} \frac{g(n) \varrho_{\varepsilon}\left(h_{1}, h_{2} ; n\right)}{n} \leqslant \gamma_{5}^{\prime \prime}(X)=\sum_{n \leqslant X} \frac{g_{5}^{\prime \prime}(n)}{n} .
$$

Démonstration. Désignons par $\left(a_{k}\right)_{k \geqslant 1}$ la suite majorante de $\left(g\left(p^{k}\right) \varrho_{\varepsilon}\left(h_{1}, h_{2} ; p^{k}\right)\right)$ qui est fournie par la proposition 5.1 , et par $\left(b_{k}\right)_{k \leqslant 1}$ la suite $\left(g_{5}\left(p^{k}\right)\right)_{k \geqslant 1}$.

Posons

$$
A(p, \alpha)=\sum_{k=0}^{\alpha} \frac{g\left(p^{k}\right) Q_{z}\left(h_{1}, h_{2} ; p^{k}\right)}{p^{k}} \quad \text { et } \quad B(p, \alpha)=\sum_{k=0}^{\alpha} \frac{g_{5}^{\prime \prime}\left(p^{k}\right)}{p^{k}}
$$

D'après le lemme 6.2, il suffit, pour démontrer la présente proposition, de prouver que l'on a, pour tout $p \geqslant 5$ et tout $\alpha \in N, A(p, \alpha) \leqslant B(p, \alpha)$, ce qui se fera en prouvant que

$$
1+\sum_{k=1}^{\alpha} \frac{a_{k}}{p^{k}} \leqslant 1+\sum_{k=1}^{\alpha} \frac{b_{k}}{p^{k}}
$$

Dans le cas $p \geqslant 5, p \nmid h_{1}^{2}+h_{2}^{2}$, on a

$$
\left(a_{k}\right)=(5,9,14,19,24, \ldots, 5 k-1, \ldots)
$$

$$
\left(b_{k}\right)=\left(5,15,35,70,126, \ldots, \frac{(k+1)(k+2)(k+3)(k+4)}{24}, \ldots\right)
$$

et l'inégalité entre les sommes à considérer est en fait vérifiée terme à terme Dans le cas $p \geqslant 5, p \mid h_{1}^{2}+h_{2}^{2}$, on a

$$
\left(a_{k}\right)=\left(2,5,4,5 p, 14 p, 9,5 p^{2}, 24 p^{2}, 14,5 p^{3}, 34 p^{3}, \ldots\right)
$$

"le" terme général étant $\left(5 k^{\prime}-0,5\right) p^{k^{\prime}}\left(\right.$ si $\left.k=2 k^{\prime}\right),\left(10 k^{\prime}+4\right) p^{k^{\prime}}\left(\right.$ si $\left.k=2 k^{\prime}+1\right)$, et

$$
\left(b_{k}\right)=\left(7,25,65,140,266,462,750, \ldots, \frac{(k+1)(k+2)(k+3)(3 k+4)}{24}, \ldots\right) .
$$


Il suffit alors de vérifier la suite d'inégalités

$$
\begin{aligned}
1+\frac{2,5}{p} & \leqslant 1+\frac{7}{p} \\
1+\frac{7}{p} & \leqslant 1+\frac{7}{p}+\frac{25}{p^{2}} \\
1+\frac{7}{p}+\frac{14}{p^{2}} & \leqslant 1+\frac{7}{p}+\frac{25}{p^{2}}+\frac{65}{p^{3}} \\
1+\frac{7}{p}+\frac{23,5}{p^{2}} & \leqslant 1+\frac{7}{p}+\frac{25}{p^{2}}+\frac{65}{p^{3}}+\frac{140}{p^{4}}, \\
1+\frac{7}{p}+\frac{23,5}{p^{2}}+\frac{24}{p^{3}} & \leqslant 1+\frac{7}{p}+\frac{25}{p^{2}}+\frac{65}{p^{3}}+\frac{140}{p^{4}}+\frac{266}{p^{5}}
\end{aligned}
$$

etc...

Les membres de gauche sont les sommes tronquées d'une série de terme général $(15 k-6,5) / p^{k}$, et les membres de droite les sommes tronquées d'une série de terme général $(k+1)(k+2)(k+3)(3 k+4) / 24 p^{k}$. L'inégalité est donc vérifiée terme à terme (et de plus les sommes de gauche sont tronquées "plus court" que celles de droite).

Les prochains résultats seront des majorations des sommes

$$
G_{0}^{\prime \prime}(X)=\sum_{n \leqslant X} g_{0}^{\prime \prime}(n) \quad \text { et } \quad \gamma_{0}^{\prime \prime}(X)=\sum_{n \leqslant X} \frac{g_{0}^{\prime \prime}(n)}{n},
$$

mais il faut auparavant estimer le produit $\Pi(1+2 / p)$ pour les nombres premiers $p \geqslant 5$ dont le carré divise $h_{1}^{2}+h_{2}^{2}$.

Lemme 6.4. Les entiers (non nuls) $h_{1}$ et $h_{2}$ étant fixés et vérifiant $\left|h_{1}\right|+\left|h_{2}\right| \leqslant P$, on $a$ :

$$
\prod_{\substack{p^{2} \mid h^{2}+h^{2} \\ p \geqslant 5}}\left(1+\frac{2}{p}\right) \leqslant K_{p}=0,297621(\log \log P)^{2} .
$$

Démonstration. On a $1+2 / p<\exp (2 / p)$ et il suffira d'utiliser une majoration de $\sum_{p^{2} \mid k_{1}^{2}+h_{2}^{2}}(1 / p)$, laquelle résultera elle-même d'une majoration du nombre des nombres premiers $p \geqslant 5$ dont le carré divise $h_{1}^{2}+h_{2}^{2}$. On a card $\left\{p \mid p^{2}\right.$ divise $\left.h_{1}^{2}+h_{2}^{2}\right\} \leqslant \max \left\{t \mid\left(2 \cdot 3 \cdot 5 \cdot \ldots \cdot p_{t}\right)^{2} \leqslant h_{1}^{2}+h_{2}^{2}\right\}$

$$
\leqslant \max \left\{t \mid 2 \cdot 3 \cdot 5 \cdot \ldots \cdot p_{t} \leqslant P\right\} \text {. }
$$

On dispose de la minoration, valable pour $t \geqslant 13$ ([7], p. 74):

$$
t \log t<\vartheta\left(p_{t}\right)=\sum_{k=1}^{t} \log p_{k} .
$$

Si on désigne alors par $T$ le plus grand entier qui satisfait

$$
T \log T \leqslant \log P,
$$

on a, sous la condition $P \geqslant 3,05 \cdot 10^{14}$ (qui implique $T \geqslant 13$ ),

$$
\text { card }\left\{p \mid p^{2} \text { divise } h_{1}^{2}+h_{2}^{2}\right\} \leqslant T \text {. }
$$

Il faut maintenant majorer la somme des $1 / p$, pour laquelle on dispose de la majoration, valable pour tout $x$ ([8]):

$$
\sum_{p \leqslant x} \frac{1}{p}<\log \log x+B+\frac{1}{\log ^{2} x}
$$

avec $B=0,2614972 \ldots$ On dispose enfin de l'encadrement, valable pour $n \geqslant 6$ (ibid.):

$$
n \log n<p_{n}<(\log n+\log \log n) .
$$

On peut maintenant écrire, sous la même condition $P \geqslant 3,05 \cdot 10^{14}$,

$$
\begin{aligned}
\sum_{\substack{p^{2} \mid h_{1}^{2}+h_{2}^{2} \\
p \leqslant 5}} \frac{1}{p} & \leqslant \sum_{k=3}^{T+2} \frac{1}{p_{k}}=\left(\sum_{k=1}^{T} \frac{1}{p_{k}}\right)-\frac{5}{6}+\frac{1}{p_{T+1}}+\frac{1}{p_{T+2}} \\
& \leqslant\left(\sum_{\substack{p \leqslant T(\log T+\log \log T) \\
p}} \frac{1}{p}\right)-\frac{5}{6}+\frac{2}{T(\log T+\log \log T)} \\
& \leqslant f(T),
\end{aligned}
$$

avec

$$
\begin{aligned}
f(T)=\log \log \{T(\log T+\log \log T)\}+B-\frac{5}{6} \\
+\frac{1}{\log ^{2}\{T(\log T+\log \log T)\}}+\frac{2}{T(\log T+\log \log T)}
\end{aligned}
$$

Pour obtenir la meilleure majoration du reste, il faut exclure les "petites" valeurs de $T$. On suppose donc maintenant $P \geqslant 10^{80}$, ce qui implique $T \geqslant 47$, $\log T \geqslant 3,850147, T \log T \geqslant 180,956, T(\log T+\log \log T) \geqslant 244,318$. Par ail- 
leurs on a la suite d'inégalités

$$
\begin{aligned}
& \log \log \{T(\log T+\log \log T)\}=\log \log \left\{T \log T\left(1+\frac{\log \log T}{T \log T}\right)\right\} \\
& <\log \left(\log (T \log T)+\frac{\log \log T}{T \log T}\right) \\
& =\log \{\log (T \log T) \times \\
& \left.\times\left(1+\frac{\log \log T}{T \log T \log (T \log T)}\right)\right\} \\
& <\log \log (T \log T)+\frac{\log \log T}{T \log T \log (T \log T)} .
\end{aligned}
$$

Comme $T \log T \leqslant \log P$, on obtient en définitive

$$
f(T)<\log \log \log P+0,0014332+B-\frac{5}{6}+0,0330763+0,0081861
$$

$$
<\log \log \log P-0,529140 \text {. }
$$

On peut écrire, toujours sous la condition $P \geqslant 10^{80}$ (qui implique $T+2 \geqslant 49$, et donc $P_{T+2} \geqslant 227$ ),

$$
\begin{aligned}
C_{h_{1}^{2}+h_{2}^{2}} & =\prod_{\substack{p^{2} \mid h_{1}^{2}+h_{2}^{2} \\
p \geqslant 5}}\left(1+\frac{2}{p}\right) \leqslant \prod_{k=3}^{T+2}\left(1+\frac{2}{p_{k}}\right) \\
& \leqslant \prod_{k=3}^{49}\left(1+\frac{2}{p_{k}}\right) \exp \left(2 \sum_{k=50}^{T+2} \frac{1}{p_{k}}\right) \\
& =\prod_{k=3}^{49}\left\{\left(1+\frac{2}{p_{k}}\right) \exp \left(-\frac{2}{p_{k}}\right)\right\} \exp \left(2 \sum_{k=3}^{T+2} \frac{1}{p_{k}}\right) \\
& \leqslant 0,857568 \exp (2 \log \log \log P-1,058280) \\
& =0,297621(\log \log P)^{2} .
\end{aligned}
$$

Corollaire (de la proposition 6.4). Les entiers (non nuls) $h_{1}$ et $h_{2}$ étant fixés et vérifiant $\left|h_{1}\right|+\left|h_{2}\right| \leqslant P$, la fonction $g_{0}^{\prime \prime}(n)=g_{0}^{\prime \prime}\left(h_{1}, h_{2} ; n\right)$ étant celle définie dans l'énoncé de la proposition 6.3, on a pour $P \geqslant 10^{80}$ la majoration

$$
\gamma_{0}^{\prime \prime}(X)=\sum_{n \leqslant X} g_{0}^{\prime \prime}(n) / n \leqslant K_{P}
$$

Démonstration. Elle est triviale: $\sum_{n \leqslant X} g_{0}^{\prime \prime}(n) / n$ est composée d'une partie des termes du développement du produit $\prod_{p^{2} \mid h_{1}^{2}+h_{2}^{2}}(1+2 / p)$, majoré
par $K_{P}$.
Proposition 6.5. Les entiers (non nuls) $h_{1}$ et $h_{2}$ étant fixés et vérifiant $\left|h_{1}\right|+\left|h_{2}\right| \leqslant P$, on a pour $P \geqslant 10^{80}$ la majoration

$$
\begin{aligned}
\gamma_{5}^{\prime \prime}(X)= & \sum_{n \leqslant X} \frac{g_{5}^{\prime \prime}(n)}{n} \\
\leqslant & \frac{1,01722}{3^{5}} K_{P}\left(\frac{1}{120} \log ^{5}\left(10^{6} X\right)\right. \\
& +\frac{5}{24} \log ^{4}\left(10^{6} X\right)+\frac{5}{3} \log ^{3}\left(10^{6} X\right) \\
& \left.+5 \log ^{2}\left(10^{6} X\right)+5 \log \left(10^{6} X\right)+1\right) .
\end{aligned}
$$

Corollaire. On a, pour $P \geqslant 10^{80}$, la majoration

$$
\sigma_{\varepsilon}^{\prime \prime}\left(h_{1}, h_{2} ; P\right)=\sum_{\substack{\delta \leqslant 7 P \\ \delta \delta, 6)=1}} \frac{g(\delta) \varrho_{\varepsilon}\left(h_{1}, h_{2} ; \delta\right)}{\delta} \leqslant 5,94321 \cdot 10^{-5} K_{P}(\log P)^{5} .
$$

Démonstration de la proposition. En respectant les conventions de notations adoptées jusqu'ici, posons

$$
l_{0}=g_{0}^{\prime \prime}, \quad l_{j}=l_{j-1} * i \quad(j=1,2, \ldots, 5),
$$

de sorte que $I_{5}=d_{5}^{\prime} * g_{5}^{\prime \prime}$, où $d_{5}^{\prime}$ est la fonction de diviseurs $d_{5}$ restreinte aux facteurs locaux pour $p=2$ et $p=3$. Posons enfin

$$
L_{j}(X)=\sum_{n \leqslant X} l_{j}(n) \quad \text { et } \quad \lambda_{j}(X)=\sum_{n \leqslant X} l_{j}(n) / n .
$$

Dans un premier temps, on calcule de proche en proche des majorations de $L_{j}(X)$ et de $\lambda_{j}(X)$, en utilisant les formules de récurrence suivantes:

$$
L_{j}(X)=\sum_{n \leqslant X}\left(l_{j-1} * i\right)(n)=\sum_{n \leqslant X} l_{j-1}(n)\left[\frac{X}{n}\right] \leqslant X \lambda_{j-1}(X),
$$

et

$$
\lambda_{j}(X)=\int_{1}^{X} \frac{d L_{j}(t)}{t}=\frac{L_{j}(X)}{X}+\int_{i}^{X} \frac{L_{j}(t)}{t^{2}} d t .
$$

- majoration initiale $\lambda_{0}(X)=\gamma_{0}^{\prime \prime}(X) \leqslant K_{P}$;

- majoration de $L_{1}(X)$ :

$$
K_{P} X
$$

- majoration de $\lambda_{1}(X)$

$$
K_{P}+\int_{1}^{x} \frac{K_{P} t}{t^{2}} d t=K_{P}(\log X+1)
$$

- majoration de $L_{2}(X)$ :

$$
K_{p} X(\log X+1)
$$


- majoration de $\lambda_{2}(X)$ :

$$
K_{P}(\log X+1)+\int_{1}^{X} \frac{K_{P} t(\log t+1)}{t^{2}} d t=K_{P}\left(\frac{1}{2} \log ^{2} X+2 \log X+1\right)
$$

- majoration de $L_{3}(X)$ :

$$
K_{P} X\left(\frac{1}{2} \log ^{2} X+2 \log X+1\right)
$$

- majoration de $\lambda_{3}(X)$ :

$K_{p}\left(\frac{1}{2} \log ^{2} X+2 \log X+1\right)+\int_{1}^{x} \frac{K_{P} t\left(\frac{1}{2} \log ^{2} t+2 \log t+1\right)}{t^{2}} d t$

$=K_{P}\left(\frac{1}{6} \log ^{3} X+\frac{3}{2} \log ^{2} X+3 \log X+1\right)$;

- majoration de $L_{4}(X)$ :

$$
K_{P} X\left(\frac{1}{6} \log ^{3} X+\frac{3}{2} \log ^{2} X+3 \log X+1\right)
$$

- majoration de $\lambda_{4}(X)$ :

$K_{P}\left(\frac{1}{6} \log ^{3} X+\frac{3}{2} \log ^{2} X+3 \log X+1\right)+\int_{1}^{X} \frac{K_{P} t\left(\frac{1}{6} \log ^{3} t+\frac{3}{2} \log ^{2} t+3 \log t+1\right)}{t^{2}} d t$

$=K_{P}\left(\frac{1}{24} \log ^{4} X+\frac{2}{3} \log ^{3} X+3 \log ^{2} X+4 \log X+1\right) ;$

- majoration de $L_{5}(X)$ :

$$
K_{P} X\left(\frac{1}{24} \log ^{4} \dot{X}+\frac{2}{3} \log ^{3} X+3 \log ^{2} X+4 \log X+1\right)
$$

- majoration de $\lambda_{5}(X)$ :

$$
\begin{gathered}
K_{P}\left(\frac{1}{24} \log ^{4} X+\frac{2}{3} \log ^{3} X+3 \log ^{2} X+4 \log X+1\right) \\
\quad+\int_{1}^{x} \frac{K_{P} t\left(\frac{1}{24} \log ^{4} t+\frac{2}{3} \log ^{3} t+3 \log ^{2} t+4 \log t+1\right)}{t^{2}} d t \\
=K_{P}\left(\frac{1}{120} \log ^{5} X+\frac{5}{24} \log ^{4} X+\frac{5}{3} \log ^{3} X+5 \log ^{2} X+5 \log X+1\right) .
\end{gathered}
$$

On utilise alors le produit de convolution $l_{5}=d_{5}^{\prime} * g_{5}^{\prime \prime}$ pour écrire, $A$ étant un réel qui sera fixé ultérieurement,

soit

$$
\sum_{n \leqslant A X} \frac{l_{5}(n)}{n} \geqslant\left(\sum_{\substack{n^{\prime}=2^{a_{3} b} \\ n^{\prime} \leqslant A}} \frac{d_{5}^{\prime}\left(n^{\prime}\right)}{n^{\prime}}\right)\left(\sum_{n^{\prime \prime} \leqslant X} \frac{g_{5}^{\prime \prime}\left(n^{\prime \prime}\right)}{n^{\prime \prime}}\right),
$$

$$
\gamma_{s}^{\prime \prime}(X) \leqslant\left(\sum_{\substack{n^{\prime}=2^{a_{3}} \\ n^{\prime} \leqslant A}} \frac{d_{5}^{\prime}(n)}{n^{\prime}}\right)^{-1} \lambda_{5}(A X)
$$

Des essais numériques montrent que les valeurs numériques optimales (par rapport à la contrainte $P \geqslant 10^{80}$ et en vue du corollaire) sont obtenues pour $A$ entre $10^{6}$ et $10^{7}$. On calcule sans difficulté

$$
\sum_{\substack{n^{\prime}=2^{a_{3} b} \\ n^{\prime} \leqslant 10^{6}}} \frac{d_{5}^{\prime}\left(n^{\prime}\right)}{n^{\prime}}=0,983076 \ldots \times 3^{5}>3^{5} / 1,01722,
$$

et cela termine la démonstration de la proposition.

Démonstration du corollaire. Pour $X=7 P \geqslant 7 \cdot 10^{80}$, on a

$\frac{1}{120} \log ^{5}\left(10^{6} X\right)+\frac{5}{24} \log ^{4}\left(10^{6} X\right)+\frac{5}{3} \log ^{3}\left(10^{6} X\right)+5 \log ^{2}\left(10^{6} X\right)$

$$
\begin{array}{r}
+5 \log \left(10^{6} X\right)+1 \\
=\frac{1}{120} \log ^{5} P\left(\frac{\log ^{5}\left(7 \cdot 10^{6} P\right)}{\log ^{5} P}+25 \frac{\log ^{4}\left(7 \cdot 10^{6} P\right)}{\log ^{5} P}+\ldots\right) \\
\leqslant \frac{1}{120} \log ^{5} P\left(\frac{a^{5}+25 a^{4}+200 a^{3}+600 a^{2}+600 a+120}{b^{5}}\right),
\end{array}
$$

en notant $a=\log \left(7 \cdot 10^{86}\right)$ et $b=\log 10^{80}$. On considère alors les valeurs

$$
\begin{gathered}
\frac{1,01722}{3^{5} \times 120}, \\
\frac{a^{5}+25 a^{4}+200 a^{3}+600 a^{2}+600 a+120}{b^{5}}=1,70369 \ldots,
\end{gathered}
$$

et l'on obtient

$$
\gamma_{5}^{\prime \prime}(7 P) \leqslant 5,94321 \cdot 10^{-5} K_{P}(\log P)^{5},
$$

cependant que l'on rappelle (proposition 6.3) que $\gamma_{5}^{\prime \prime}(7 P)$ majore $\sigma_{\varepsilon}^{\prime \prime}(P)$.

Proposition 6.6. On a, pour $P \geqslant 10^{80}$, la majoration

$$
M_{\varepsilon}\left(r_{1}, r_{2}\right) \leqslant 3,95893 \cdot 10^{-4} \mu_{\varepsilon}\left(r_{1}, r_{2}\right) P(\log P)^{5}(\log \log P)^{2} .
$$

Démonstration. On reporte dans la majoration (5.2) la valeur $2 C$ $=3,19736 \ldots$ (proposition 4.2), la majoration en $\mu_{\varepsilon}\left(r_{1}, r_{2}\right) \sigma_{\varepsilon}^{\prime \prime}\left(h_{1}, h_{2} ; P\right)$ de la proposition 6.1, et la majoration de $\sigma_{c}^{\prime \prime}\left(h_{1}, h_{2} ; P\right)$ du corollaire de la proposition 6.5. On trouve

$$
M_{\varepsilon}\left(r_{1}, r_{2}\right) \leqslant 1,33019 \cdot 10^{-3} \mu_{\varepsilon}\left(r_{1}, r_{2}\right) K_{P} P(\log P)^{5},
$$

et il ne reste plus qu'à reporter la valeur de $K_{P}$ (lemme 6.4).

6b. Sommation des constantes et démonstration du théorème principal. En conjuguant les résultats des propositions 3.5 et 6.6 , on obtient, pour $P$ 
$\geqslant 10^{80}$, la majoration

$$
H_{\varepsilon}(P) \leqslant 1,62317 \cdot 10^{-3}\left(\sum_{\substack{r_{1} \bmod 6 \\ r_{2} \bmod 6}} C_{\varepsilon}\left(r_{1}, r_{2}\right)\right) P^{3}(\log P)^{9}(\log \log P)^{2},
$$

avec $C_{\varepsilon}\left(r_{1}, r_{2}\right)=\gamma_{\varepsilon}\left(r_{1}, r_{2}\right) \delta\left(r_{1}\right) \delta\left(r_{2}\right) \mu_{\varepsilon}\left(r_{1}, r_{2}\right)$ : les coefficients $\gamma_{\varepsilon}\left(r_{1}, r_{2}\right)$ sont donnés par les tableaux qui accompagnent la proposition 2.1, les $\delta\left(r_{i}\right)$ par la liste qui accompagne le corollaire de la proposition 3.3, et enfin les $\mu_{\varepsilon}\left(r_{1}, r_{2}\right)$ par les tableaux qui accompagnent la proposition 6.1.

En effectuant les produits, on obtient les tableaux suivants:

\begin{tabular}{|c|c|c|c|c|}
\hline \multicolumn{5}{|c|}{$C_{0}\left(r_{1}, r_{2}\right)$} \\
\hline$r_{1}$ & 0 & 1 ou 5 & 2 ou 4 & 3 \\
\hline 0 & 6,46894 & 0,05024 & 0,27255 & 1,17556 \\
\hline 1 ou 5 & 0,05024 & 0,00037 & 0,01078 & 0,00170 \\
\hline 2 ou 4 & 0,27255 & 0,01078 & 0,05848 & 0,04953 \\
\hline 3 & 1,17556 & 0,00170 & 0,04953 & 0,03970 \\
\hline
\end{tabular}

\begin{tabular}{|c|c|c|c|c|}
\hline \multicolumn{5}{|c|}{$C_{1}\left(r_{1}, r_{2}\right)$} \\
\hline$r_{\mathrm{t}}$ & 0 & 1 ou 5 & 2 ou 4 & 3 \\
\hline 0 & 0,36106 & 0,00335 & 0,01522 & 0,01135 \\
\hline 1 ou 5 & $0,00335-$ & 0,00011 & 0,00072 & 0,00049 \\
\hline 2 ou 4 & 0,01522 & 0,00072 & 0,00327 & 0,00331 \\
\hline 3 & 0,01135 & 0,00049 & 0,00331 & 0,01135 \\
\hline
\end{tabular}

On en déduit:

$$
\sum_{\substack{r_{1} \bmod 6 \\ r_{2} \bmod 6}} C_{0}\left(r_{1}, r_{2}\right)=10,67748 \text { et } \sum_{\substack{r_{1} \bmod 6 \\ r_{2} \bmod 6}} C_{1}\left(r_{1}, r_{2}\right)=0,50387,
$$

d'où les produits $0,017331 \ldots$ et $0,8178 \ldots \cdot 10^{-3}$ qui fournissent les constantes du théorème principal.

\section{Bibliographie}

[1] R. Balasubramanian, J.-M. Deshouillers et F. Dress, Problème de Waring pour les bicarrés, 1: schéma de la solution, C. R. Acad. Sci. Paris 303 (1986), p. 85-88.

[2] _ - - Problème de Waring pour les bicarrés, 2: résultats auxiliaires pour le théorème asymptotique, ibid. 303 (1986), p. 161-163.
[3] J. G. van der Corput, Une inégalité relative au nombre des diviseurs, Koniklijke Nederlandsche Akad. Wet. Proceedings, 42 (1939), p. 547-553.

[4] J.-M. Deshouillers, Problème de Waring pour les bicarrés, Sem. Th. Nbs Bordeaux, année 1984-1985, exp. 14.

[5] P. Erdôs, On the sum $\sum_{1 \leqslant k \leqslant x} d(f(k))$, J. London Math. Soc. 27 (1952), p. 7-15.

[6] G. H. Hardy, J. E. Littlewood and G. Pólya, Inequalities, 2nd ed., Cambridge University Press, 1964.

[7] G. Robin, Grandes valeurs de fonctions arithmétiques et problèmes d'optimisation en nombres entiers, Thèse de Doctorat d'Etat, Limoges, 1983.

[8] J.-B. Rosser and L. Schoenfeld, Approximate formulas for some functions on prime numbers. Illinois J. Math. 6 (1962), p. 64-94.

[9] D. Wolke, A new proof of a theorem of van der Corput, J. London Math. Soc. 5 (1972), p. 609-612.

CENTRE DE RECHERCHES EN MATHÉMATIQUES DE BORDEAUX (UA 040226) UNIVERSITÉ BORDEAUX

351 . Cours de lal Liberialton

Reçu le 30.4.1986

et dans la forme modifiée le 15.9 .1986 